\title{
Variabilité des débitages laminaires au Second Mésolithique et au Néolithique ancien dans le nord de la France (VIIe et VIe millénaire BCE)
}

\author{
Pierre Allard \\ CNRS, UMR-7055, Préhistoire et Technologie, Maison de l’Archéologie et de l’Ethnologie, 21 allée de \\ l’Université, 92023, Nanterre Cedex, France. Email: pierre.allard@cnrs.fr
}

\section{Résumé :}

Cet article présente un bilan des techniques de taille du Second Mésolithique et du Néolithique ancien du $7^{\mathrm{e}}$ au $6^{\mathrm{e}}$ millénaire dans le nord de la France. Cette période est caractérisée par l'apparition d'une nouvelle pratique technique qui se manifeste par la production de lames régulières associées à de nouvelles armatures de flèche de forme trapézoïdale. D’après les études menées, il semble que seuls les débitages à la percussion indirecte sont documentés dans l'ouest et le nord de l'aire considérée. Néanmoins, des analyses plus fines devront être menées pour les débitages soignés des productions de lamelles régulières et des petits nucléus présents dans les contextes de l'est de la France. Il semble désormais qu'une méthode de débitage assez commune puisse se percevoir. Elle a été parfaitement décrite sur le site des Essart à Poitiers (méthode de l'Essart A, Marchand 2009). Cette méthode d'exploitation des blocs avec des flancs orthogonaux, un plan de frappe lisse peu incliné et une surface lamellaire quadrangulaire peu cintrée apparaît comme plutôt fréquente dans les assemblages à trapèzes du Mésolithique. Malgré un concept commun, l'étude de ce "complexe » des industries à trapèzes montre une certaine variabilité du point de vue des méthodes de taille. Il semble se dégager des entités parfois bien circonscrites au sein de cet ensemble. C'est le cas du Retzien qui s'oppose nettement au Téviécien de par les procédés de préparation systématique par micro-facettage mais également par une excellente unité stylistique des types de trapèzes. Le débitage sur tranche d'éclat pour l'installation des surfaces laminaires semble pour le moment cantonné d'une zone allant du Bassin parisien à la Belgique. L'est de la France se démarque par des productions avec des talons facettés et des plans très inclinés. Il faudra donc donner du sens à ces différentes pratiques qui pour le moment sont des résultats bruts.

La dimension chronologique est un élément crucial qui devra être mieux précisé. En rapport avec les résultats obtenus sur les modalités de la percussion indirecte, un meilleur cadre chronologique permettra de comprendre la synchronie ou la diachronie des variantes observées des débitages. De nouvelles découvertes et de nouvelles datations permettent de mieux préciser l'introduction de la percussion indirecte vers $6200 \mathrm{cal}$. BCE dans le Bassin parisien.

Enfin, il reste encore à mieux préciser les assemblages typologiques. Nous ne disposons pas encore suffisamment de séries qui permettent de bien comprendre l'évolution des différents types de trapèzes et triangles dérivés et si certaines méthodes de débitages sont particulières à certains types d'armatures. Malgré les imprécisions des datations et la difficulté de démêler les assemblages d'armatures, on peut retenir qu'il existe probablement des moments où certains types dominent. Ce

Published by the School of History, Classics and Archaeology, University of Edinburgh ISSN: 2055-0472. URL: http://journals.ed.ac.uk/lithicstudies/

This work is licensed under a Creative Commons Attribution 2.5 UK: Scotland License. 
travail reste totalement à faire et il est tributaire d'une meilleure résolution chronologique et des contextes des séries du Second Mésolithique

Mots-clés : industrie à trapèzes, débitage lamellaire, technologie lithique, Mésolithique, Néolithique ancien, punch, percussion indirecte

\section{Introduction}

Le cadre chrono-culturel que nous souhaitons appréhender dans cet article est définit par deux phénomènes majeurs qui traversent l'Europe d'est en ouest du $7^{\mathrm{e}}$ au $5^{\mathrm{e}}$ millénaire. Le premier est l'apparition d'une nouvelle pratique technique observée dans l'industrie de la pierre taillée et qui apparut suffisamment performante ou attrayante pour se diffuser rapidement dans toute l'Europe chez les communautés de chasseurs-collecteurs (à l'exception des îles britanniques). Elle se caractérise par la production de lames régulières associées à de nouvelles armatures de flèche de forme trapézoïdale, la blade and trapeze industry (Clark 1958; Kozlowski 2009). Ce stade est habituellement reconnu comme le Mésolithique dit récent-final ou "second Mésolithique". Le second phénomène est l'apparition des communautés agro-pastorales sédentaires, c'est-à-dire le Néolithique. Nous proposons dans cet article de faire un bilan des acquis récents pour les industries lithiques du VII ${ }^{\mathrm{e}}$ au VI $\mathrm{I}^{\mathrm{e}}$ millénaire BCE. La problématique que nous souhaitons développer concerne les modes de débitage observés dans ces deux grands complexes que sont les industries à trapèzes du second Mésolithique et celui du Néolithique ancien. Il sera question de l'évolution des pratiques techniques dans l'aire d'expansion terminale de ces deux grands courants, c'est-àdire principalement le nord de la France. Ce bilan fait écho à plusieurs programmes de recherche, malheureusement encore en grande partie inédits en raison des délais de publication (Perrin et al. sous presse; Allard et al. sous presse) et nous l'avons enrichi avec des découvertes récentes qui permettent de construire un meilleur cadre chronologique.

\section{Le second Mésolithique}

Faire un résumé des connaissances concernant la fin du Mésolithique est un exercice complexe car les positions scientifiques sur ces sociétés sont divergentes. Les uns voient ces groupes comme de petites unités mobiles couvrant un territoire plus ou moins vaste, les autres comme une société complexe à multiples visages dont les mutations vont faciliter l'adoption d'une économie de production (voir historique dans Valdeyron 2008; Marchand 2014a). Cette période est parfois regroupée sous le terme de «techno complexe de composant castelnovien » avec lamelles denticulées, trapèzes asymétriques et variantes, trapèzes symétriques et débitage Montbani (Kozlowski 2009).

Certaines régions sont bien pourvues de vestiges de la fin du Mésolithique (par exemple le Nord de la France et la Belgique, Rozoy 1978). Dans ces régions (comme le Tardenois), on connaît de grandes concentrations de vestiges mais qui s'avèrent de véritables palimpsestes, accumulant pendant plusieurs siècles des témoins des passages répétés de ces populations. Des sépultures sont également connues et même des nécropoles ainsi que des structures fossoyées (Verjux 2015). La taille du site serait alors le reflet de sa fréquentation répétée et pourrait signifier une organisation territoriale intégrant des camps de base ou des lieux singuliers de rassemblement, du moins pour les assemblages funéraires (Marchand 1999, 2014b).

Un problème important concerne la chronologie de cette période car les datations absolues des $6^{\mathrm{e}}$ et $7^{\mathrm{e}}$ millénaires sont encore lacunaires. Les fouilles menées dans les zones sableuses du nord de l'Europe et notamment celles des palimpsestes n'ont jamais livré de 
datations fiables. Des hiatus majeurs sont encore enregistrés dans la plupart des régions, notamment à la fin de la période où souvent un décalage important de plusieurs siècles persiste entre les derniers vestiges des chasseurs collecteurs et la néolithisation.

L'industrie de la pierre taillée, souvent le seul vestige conservé, est utilisée pour la construction d'un cadre chrono-culturel. La plupart des auteurs s'accordent pour donner une valeur culturelle forte aux armatures de flèche. Ainsi, les séquences chronologiques sont construites à partir d'une typo-chronologie relative qui fut principalement basée sur l'évolution des armatures de flèche, quellle que soit la zone considérée (par exemple: Ducrocq 2001; Rozoy 1978; Thevenin 1998; Ghesquiere 2012). Un stade final (Mésolithique final) est souvent avancé pour scinder ce second Mésolithique en deux blocs qui se manifesterait par la diversification des types et la présence d'armatures dites «évoluées ", c'est-à-dire avec certaines caractéristiques techniques divergentes des trapèzes initiaux et la disparition des armatures de la fin du premier Mésolithique.

Le phénomène de la diffusion des industries à trapèzes est désormais en partie mieux perçu suite à une critique fine des datations réalisée dans le cadre de programmes récents (Perrin et al. 2010; Perrin et al. sous presse) et en partie formalisée dans une publication de synthèse, du moins pour le sud de la France (Marchand \& Perrin 2015). Les datations les plus anciennes sont à l'heure actuelle localisées dans le sud de la méditerranée et un gradient s’observe du sud vers le nord. En résumé, les industries à trapèzes semblent plus anciennes au sud, ce qui conduit à voir une origine du phénomène dans cette région (Perrin et al. 2010). A l'échelle continentale en revanche, la faible densité de dates et de gisements disponibles, notamment en Europe centrale, ne permet pas encore de bien synthétiser ce problème même si une hypothèse balkanique a été récemment proposée et qu'une origine orientale, notamment en Crimée, est de nouveau d’actualité (Kozlowski 2009; Biagi \& Starnini 2016).

Quoiqu'il en soit, cette sériation chronologique permet de constater que l'arrivée des trapèzes dans l'aire méditerranéenne représente un changement brutal du système technique qui se manifeste par l'apparition d'un package comprenant des techniques de débitages de lames régulières, des trapèzes et des lames à coche (lame Montbani). La reprise systématique des ensembles a permis de mieux caractériser les techniques et les modes de débitage du Castelnovien. La reconnaissance du débitage par pression participe des acquis les plus récents concernant la caractérisation des industries à trapèzes (Binder 1987; Binder et al. 2012; Perrin \& Binder 2014). Sans revenir sur l'historique de cette recherche, les études technologiques ont permis de reconnaître du débitage à la pression en Méditerranée dans le complexe castelnovien et ce dès la première phase d'apparition. Le débitage à la pression du complexe castelnovien semble connaître différentes modalités qui sont corrélées à la variabilité des ressources siliceuses exploitées. L'unité tient d'une part aux procédés d'entretien du plan de pression et à la faible convexité latérale de la surface lamellaire. L'enchaînement de plusieurs séquences lamellaires lorsque le volume du matériau le permet a pour conséquence l'installation d'une surface lamellaire cintrée et une meilleure régularité des produits lamellaires (Binder et al. 2012; Perrin \& Binder 2014). Le facettage des plans de frappe et des talons des produits lamellaires est systématique. Ainsi la régularité des lamelles est variable, en relation avec les volumes offerts par les matériaux ou l'absence des phases de préparation initiale, ce qui explique pourquoi l'identification formelle de cette technique n'a pas été simple (Perrin \& Binder 2014). Cette technique participe donc clairement du package originel du phénomène d'apparition des trapèzes. Il faut également retenir qu'elle est $a$ priori, dans l'état actuel des connaissances, non exclusive. La dualité des techniques de percussion est effective puisque la percussion indirecte est également systématiquement recensée dans les séries lithiques castelnoviennes (Perrin \& Binder 2014). L'expansion des industries à trapèze semble rapide, suggérant un mouvement démique au début du $\mathrm{VII}^{\mathrm{e}}$ millénaire et se diffuse 
vers le nord et l'ouest dans la seconde moitié du VII ${ }^{\mathrm{e}}$ millénaire (Perrin et al. 2010; Perrin et al. sous presse).

La question qui se pose donc naturellement pour le quart nord-ouest de l'Europe concerne l'homogénéité du phénomène. Ainsi, constatons-nous dans cette région un phénomène analogue à celui du Castelnovien et quelles sont les techniques de débitages mises en œuvre pour les industries à trapèzes ? Enfin, peut-on voir une filiation entre les modes de débitages de la fin du Mésolithique et celle du Néolithique ancien en Europe tempérée ?

\section{Les modes de débitage du Second Mésolithique}

La reconnaissance des techniques de percussion et des méthodes de débitage en France septentrionale est historiquement inscrite avec la notion de style. C'est en effet sous cette dénomination que les débitages ont été scindés sous les termes de "style Coincy » et de «style Montbani » par J. G. Rozoy qui voyait une opposition notamment (mais pas seulement) dans la régularité des produits laminaires (Rozoy 1968). Il est intéressant de constater que cet auteur relie ces débitages à la même technique de percussion : "Les débitages de Coincy et de Montbani paraissent réalisés au chasse-lame (...), Le débitage de Montbani peut aussi avoir été obtenu par pression » (Rozoy 1968: 370). C'est probablement ce constat, fruit d'une appréciation de l'époque, qui a fait intervenir la notion de style puisque le punch est évoqué pour les deux types de débitage. Des études ultérieures ont montré que le style de Coincy correspond en fait à un débitage réalisé à la pierre (Ketterer 1997; Souffi 2004; Michel 2009; Walczak 1998). Quant au style de Montbani, que Rozoy associe déjà aux industries à trapèzes du Mésolithique récent/final (Rozoy 1968; 1978), il est de plus en plus reconnu comme réalisé à la percussion indirecte, c'est ce que nous allons évoquer plus précisément. Au vu de l'étendue de l'aire prise en considération, il apparaît que les études technologiques associant une diagnose des techniques et des modes opératoires sont encore indigentes, mais les quelques régions, ou plutôt sites, bien documentés laissent percevoir différentes modalités du débitage à la percussion indirecte (Figure 1).

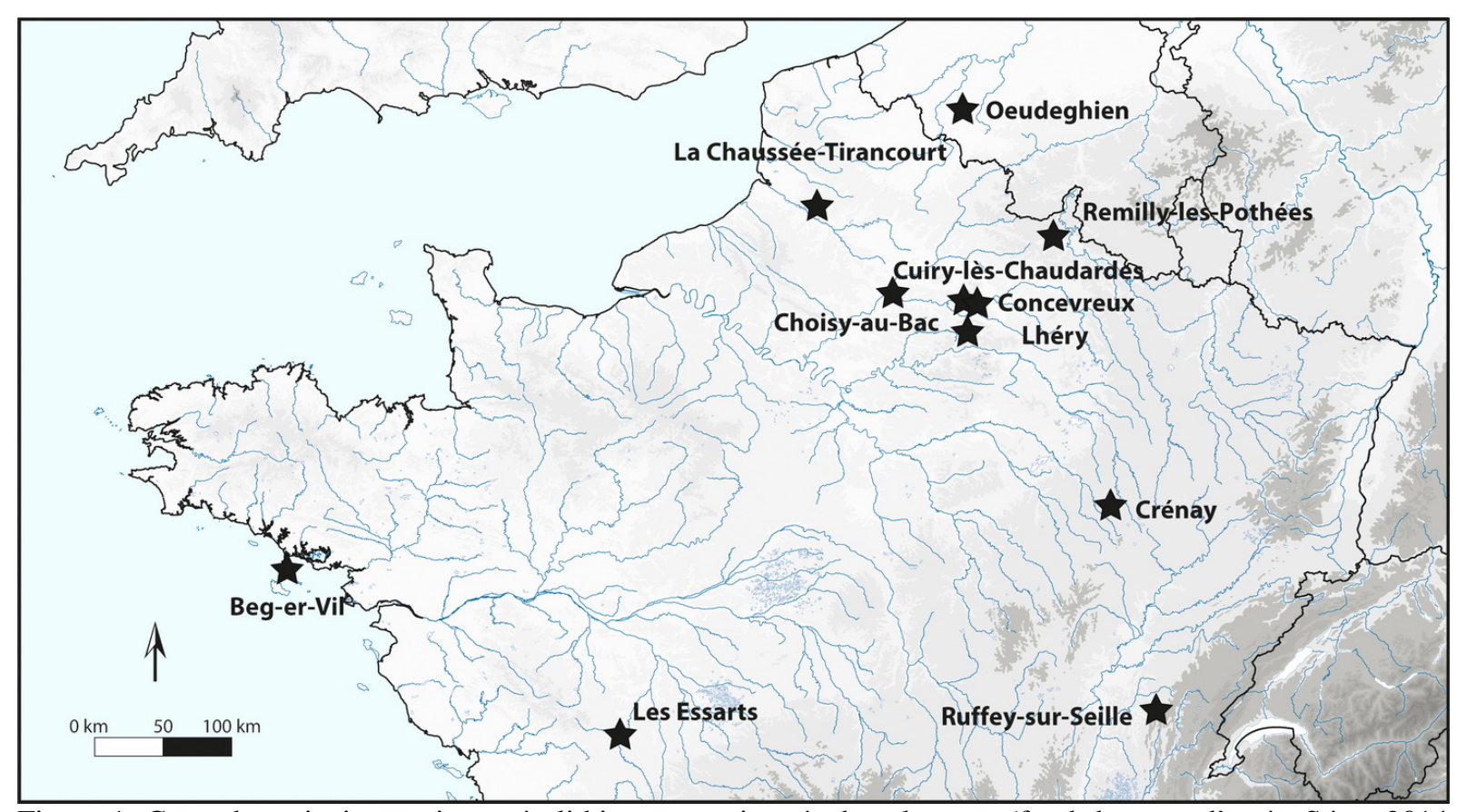

Figure 1. Carte des principaux sites mésolithiques mentionnés dans le texte (fond de carte d’après Séara 2014 modifié).

Figure 1. Map of the main Mesolithic sites mentioned in the text (background map after Séara 2014, modified) 


\subsection{Une méthode répandue}

Il semble désormais qu'une méthode de débitage assez commune puisse se percevoir. Elle a été parfaitement décrite sur le site des Essart à Poitiers (méthode de l'Essart A, Marchand 2009: 91-100).

Ainsi, une phase initiale consiste probablement à la fragmentation des blocs (suivant des plans de clivages ?) pour obtenir des petits volumes parallélépipédiques. Le volume du nucléus est circonscrit par deux flancs orthogonaux, la surface laminaire est quadrangulaire (Figure 2). Le plan de frappe est lisse, légèrement incliné et entretenu par des éclats débités depuis la surface laminaire. L'exploitation de la surface est frontale et le volume initial permet de déplacer les tables sur les flancs (Marchand 2009: 93-99). L’objectif est orienté vers une production de lamelles régulières à 3 pans de 10-12 mm de large, les corniches des talons lisses sont soigneusement abrasées. Une variante voit le développement de la surface lamellaire déborder sur les deux flancs (méthode dite Essart B, Figure 3).
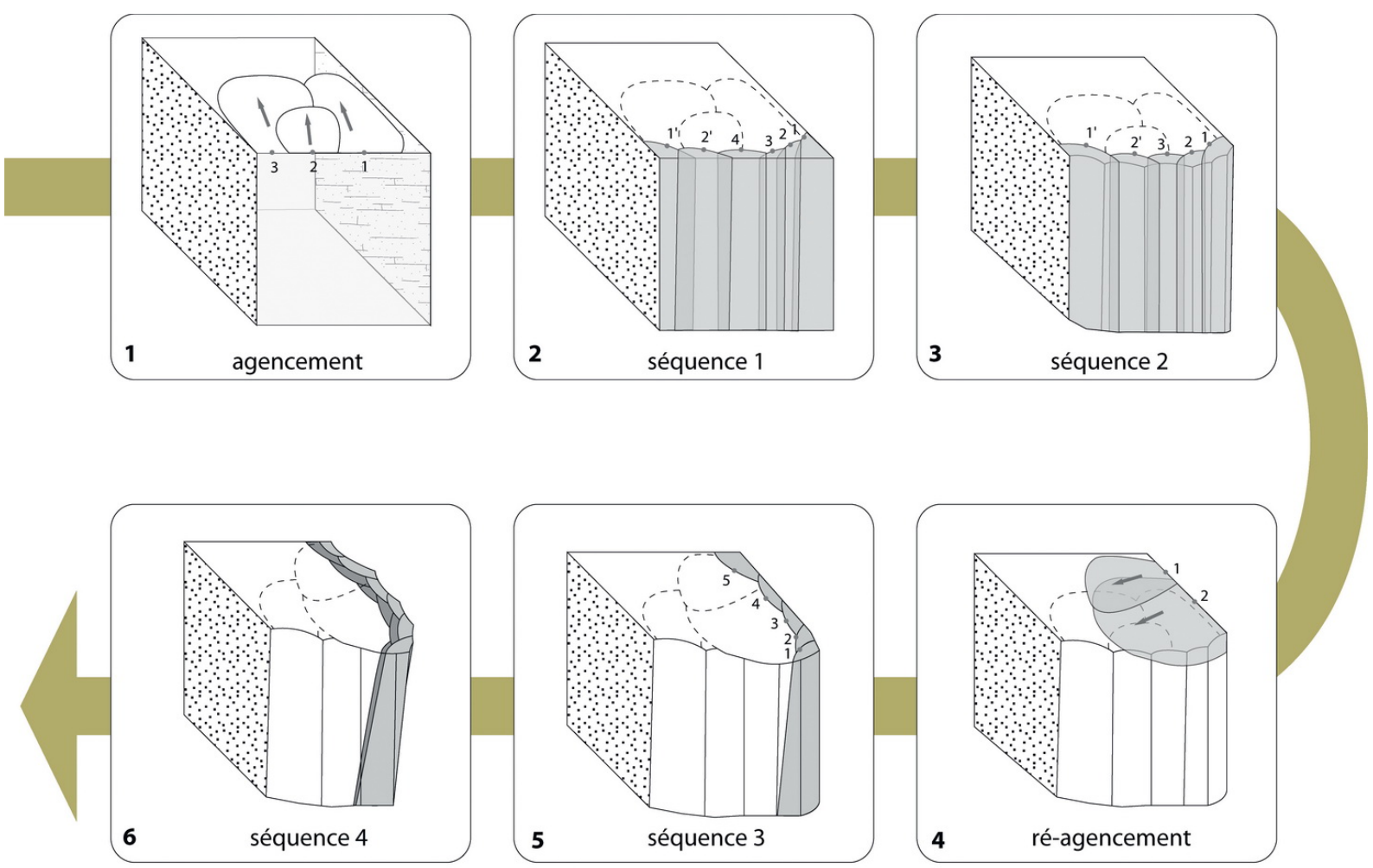

Figure 2. Schéma de débitage des séquences laminaires du second Mésolithique sur le site de l’Essart à Poitiers (Marchand 2009, 2014).

Figure 2. Diagram of the laminar sequences of the second Mesolithic on the site of Essart near Poitiers (Marchand 2009, 2014)

Les caractéristiques des produits lamellaires et des nucléus ainsi que les stigmates et les morphologies des talons indiquent pour G. marchand l'emploi de la percussion indirecte, en référence avec les travaux des expérimentateurs modernes (Marchand 2009: 106).

Cette méthode d'exploitation des blocs avec des flancs orthogonaux, un plan de frappe lisse peu incliné et une surface lamellaire quadrangulaire peu cintrée apparaît comme plutôt fréquente dans les assemblages à trapèzes du Mésolithique. L’indigence des études technologiques et la rareté des dessins des nucléus (notamment dans les fouilles anciennes) ne permettent pas de bien rendre compte de cette information. 

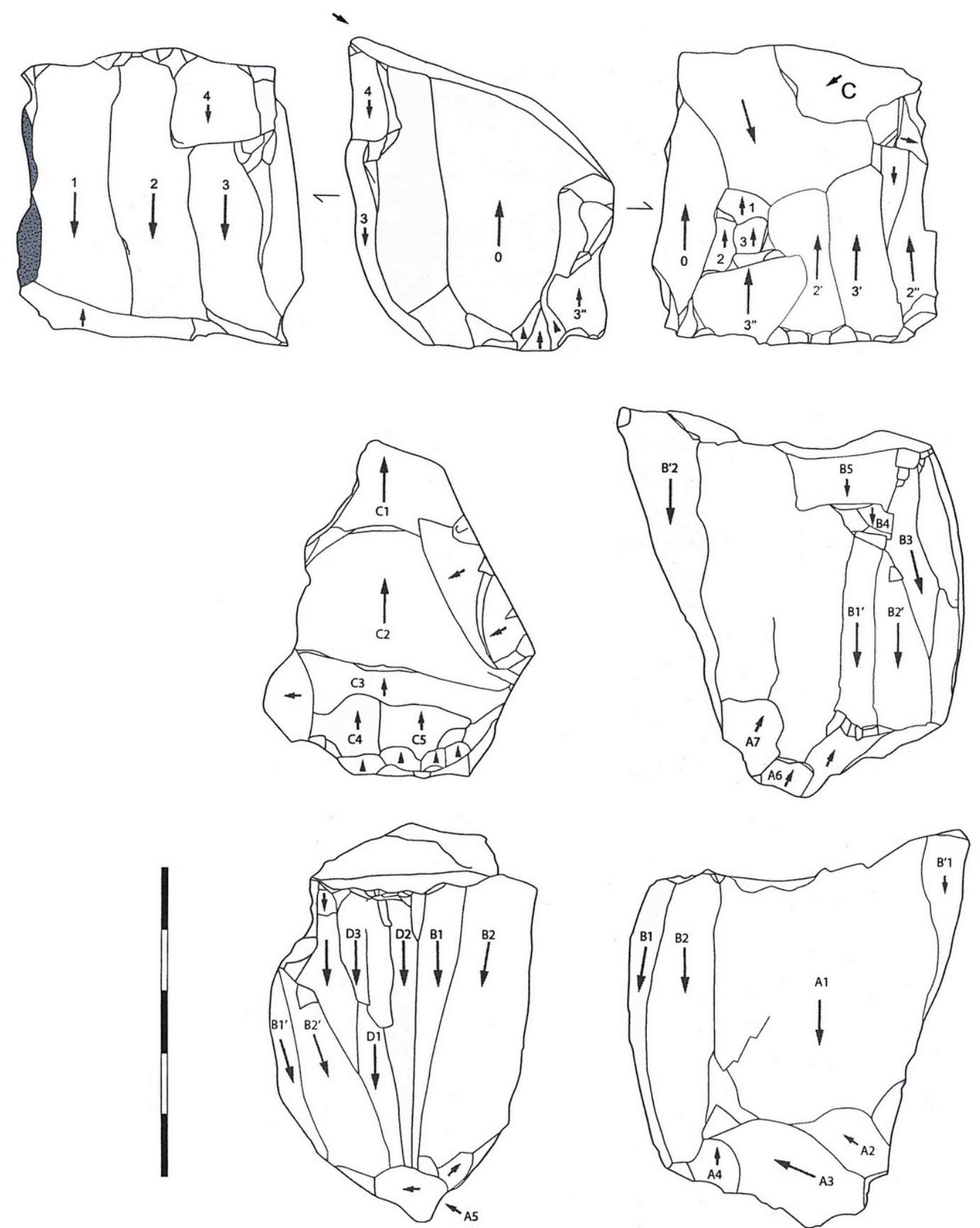

Figure 3. En haut : nucleus de méthode Essart A. En bas : nucleus de méthode Essart B (Marchand 2009: 95, 100). (L’échelle est de $5 \mathrm{~cm}$ divisée en segments de $1 \mathrm{~cm}$ )

Figure 3. Top: core of Essart method A. Bottom: core of method Essart B (Marchand 2009: 95, 100). (The scale bar is $5 \mathrm{~cm}$, divided into $1 \mathrm{~cm}$ segments.)

Néanmoins, un tour d'horizon de la bibliographie permet de les reconnaître dans la Somme, notamment à Vraignes où sont décrits des nucléus unipolaires réguliers à table plate perpendiculaire (Ducrocq 2001). Les fouilles menées dans l'Aisne et plus précisément dans le Tardenois en présentent d'après les dessins des exemplaires similaires à l'Allée Tortue (Parent 1967: 195), Montbani (Rozoy 1968), Oulchy-la-Ville (Hinout 1990) ou encore Villeneuve-sur-Fère (Hinout 1989). Les nucléus dessinés montrent en effet des flancs orthogonaux rectilignes, des plans de frappe lisses inclinés et un débitage régulier unipolaire sur une surface laminaire quadrangulaire qui semblent s'apparenter à la méthode de l'Essart A. 
La découverte récente du site de Lhéry dans la Marne apporte un éclairage supplémentaire, notamment sur les étapes de mises en forme de grands volumes (Séara \& Bostyn 2009; Bostyn \& Seara 2011). Ce site est localisé sur un gisement de silex de très bonne qualité du Bartonien. Les remontages exhaustifs de certains blocs montrent l'exploitation de larges et épaisses plaquettes qui sont mises en forme à la percussion dure puis fracturées à l'aide de pièces esquillées massives (Figure 4).

Les débitages sont unipolaires et frontaux, sur tables étroites ou peu envahissantes avec des plans de frappe lisses inclinés réalisés sur des plaquettes ou des blocs parallélépipédiques après l'étape de mise en forme. Les produits laminaires sont réguliers à talons lisses, les surfaces lamellaires sont rectilignes et l'initialisation du débitage se fait par la mise en forme d'une nervure guide par les grands éclats de préparation ou par des crêtes partielles généralement à un versant. Il s'agit donc d'un débitage proche de celui de l'Essart et la fracturation des blocs bien mise en évidence à Lhéry devait probablement avoir lieu également à l'Essart mais l'absence de remontage ne permet pas de le confirmer avec certitude sur ce dernier site.

\subsection{Une variabilité attestée}

Néanmoins à Lhéry, une part des déchets initiaux et des éclats issus de la fracturation lors de la mise en forme des grandes plaquettes font l'objet d'une exploitation plus singulière où la surface laminaire est installée sur la tranche de l'éclat, profitant ainsi des convexités latérales et longitudinales favorables du support (Bostyn \& Seara 2011). Les tables sont étroites à partie distale convergente. Les plans de frappe sont lisses. Cette variante n'est pas spécifique à Lhéry. En effet, nous avons eu l'occasion d'étudier ce type débitage sur éclat-support à l'Allée Tortue XIV (fouilles J.G Rozoy) sur du silex tertiaire probablement bartonien, mais aussi à Oeudeghien (Crombé et al. 1992) en Belgique dans un autre matériau (Figure 5).

Ce type de débitage nécessite donc la production d'éclats massifs et il est vraisemblable qu'il existe des lieux de production de ces éclats supports dont une partie serait emportée, Lhéry en serait l'exemple le mieux documenté. Il est difficile de connaître l'ampleur de ce débitage sur éclats dans les séries du Mésolithique à trapèzes, notamment dans le Tardenois car les études anciennes ne le mentionnent pas, bien que nous l'ayons observé à l'Allée Tortue par la suite.

Les grandes fouilles préventives réalisées dans l'est de la France apportent également une documentation étoffée pour le second Mésolithique. Ainsi, sur le site stratifié de plein air de Ruffey-sur-Seille "A Daupharde ", le niveau R1 livre une industrie à trapèzes et lames encochées assez abondante. L'étude technologique (Deschamps 2000; Seara et al. 2002: 134139) montre une préparation des blocs avec de gros éclats afin de dégager une arête propice ou l'installation d'une crête antérieure pour l'initialisation du débitage. Les nucléus sont unipolaires et prismatiques, rarement pyramidaux (6 contre 29). Les plans de frappe inclinés et facettés sont dominants, tout comme le facettage des talons des lamelles. La surface lamellaire est rectiligne avec le dos cortical ou aménagé par une crête dorsale ou postérolatérale. Certains exemplaires d'après les figures présentent une surface lamellaire quadrangulaire (Seara et al. 2002: fig. 82 et 83) (Figure 6). Les produits sont rectilignes avec une courbure prononcée en partie distale dans $20 \%$ des cas. Les largeurs oscillent entre 7 et $12 \mathrm{~mm}$ pour des produits entre 1 à $4 \mathrm{~mm}$ d'épaisseur.

La différence avec l'Essart A repose sur un facettage large des plans de frappe (2/3 des nucléus) et une fréquence élevée des talons avec micro-facettage (32\%). L’auteur avait proposé l'emploi de la percussion indirecte pour ces débitages du niveau R1 de Ruffey (Seara et al. 2002: 139). 


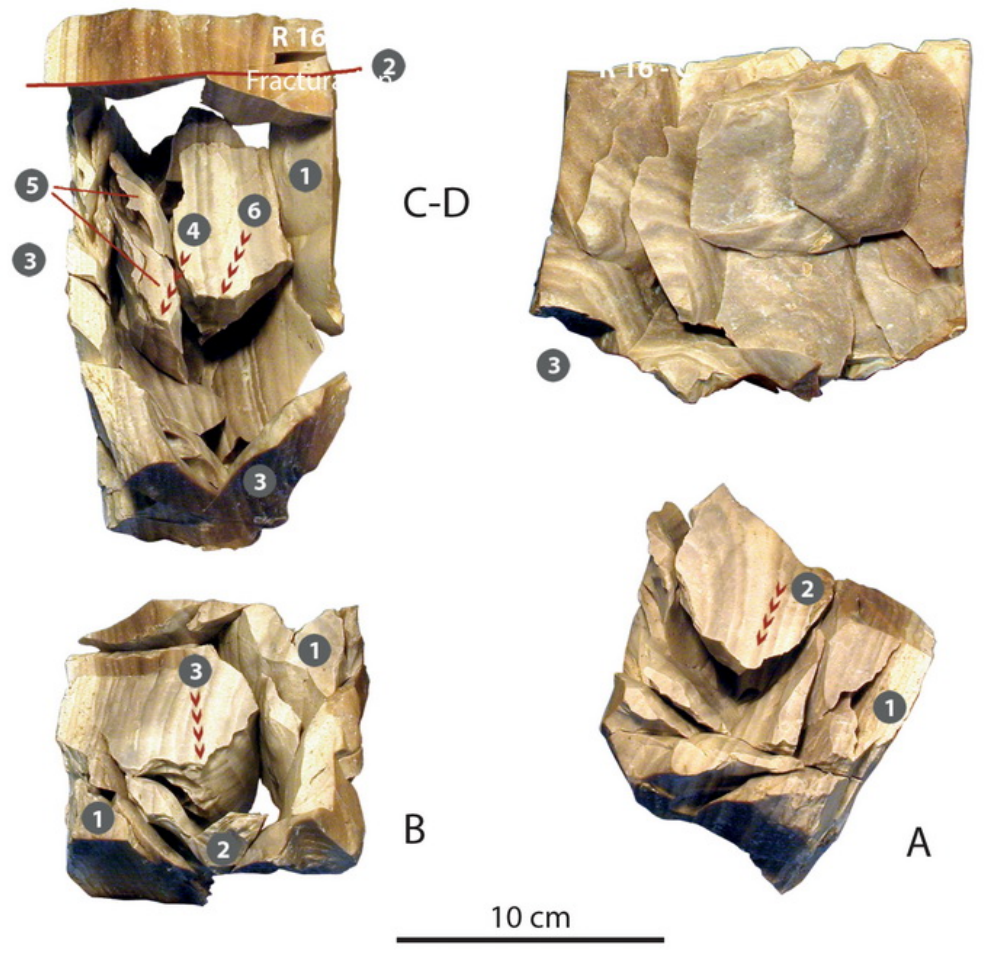

\section{Remontage 16 - C-D}

1 phase initiale d'épannelage

2 fracturation (16-D); seules les phases

d'épannelage sont représentées

3 épannelage complet du bloc

4 première phase de débitage lamellaire

5 remise en forme du flanc gauche

6 deuxième phase de débitage lamellaire

\section{Remontage 16 - B}

1 épannelage initial du bloc

2 préparation de la surface de

débitage par deux enlèvements

laminaires

3 phase de débitage lamellaire

\section{Remontage 16 - A}

1 épannelage important du bloc

2 première phase de débitage lamellaire
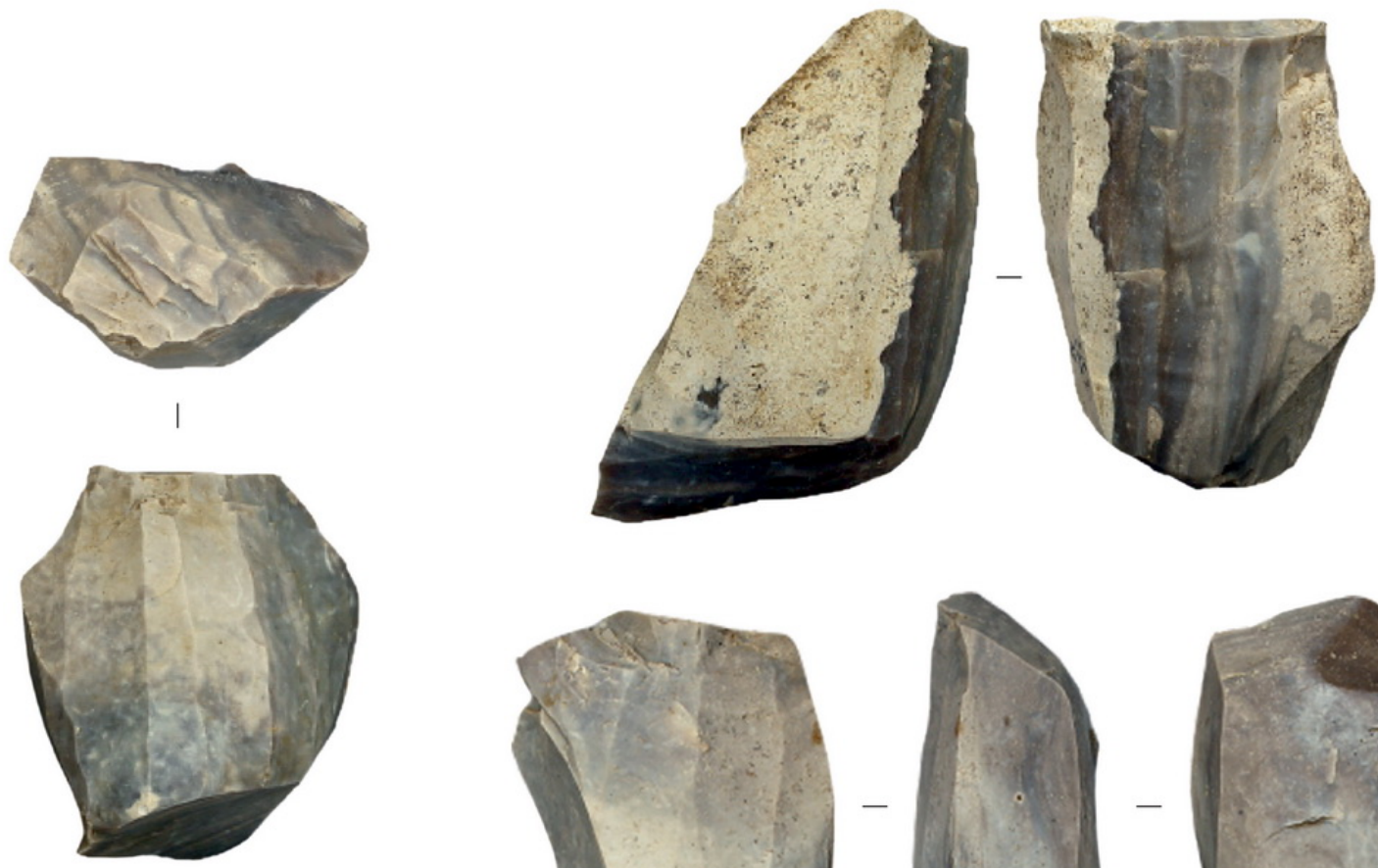

$2 \mathrm{~cm}$
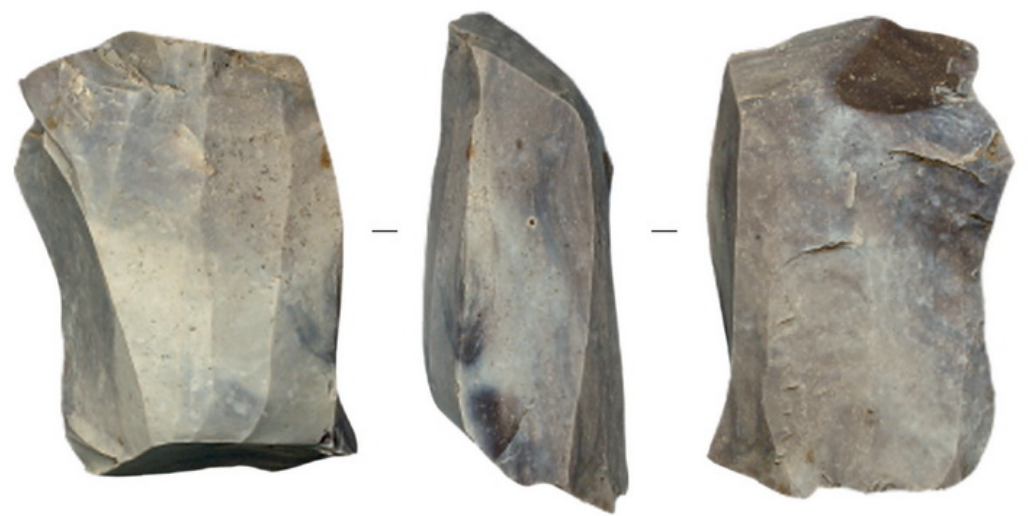

Figure 4. Lhéry (Marne). En haut: Ensemble de remontages d'une dalle en silex tertiaire (Séara 2014: 48 modifié). En bas: Exemples de nucléus à lamelles du Second Mesolithique similaires à la méthode de l'Essart $\mathrm{A}$ (d'après Séara 2014: 47).

Figure 4. Top: Refit of a tabular tertiary flint (Séara 2014: 48 modified). Below: Examples of Second Mesolithic bladelet cores similar to the Essart A method (after Séara 2014: 47). 


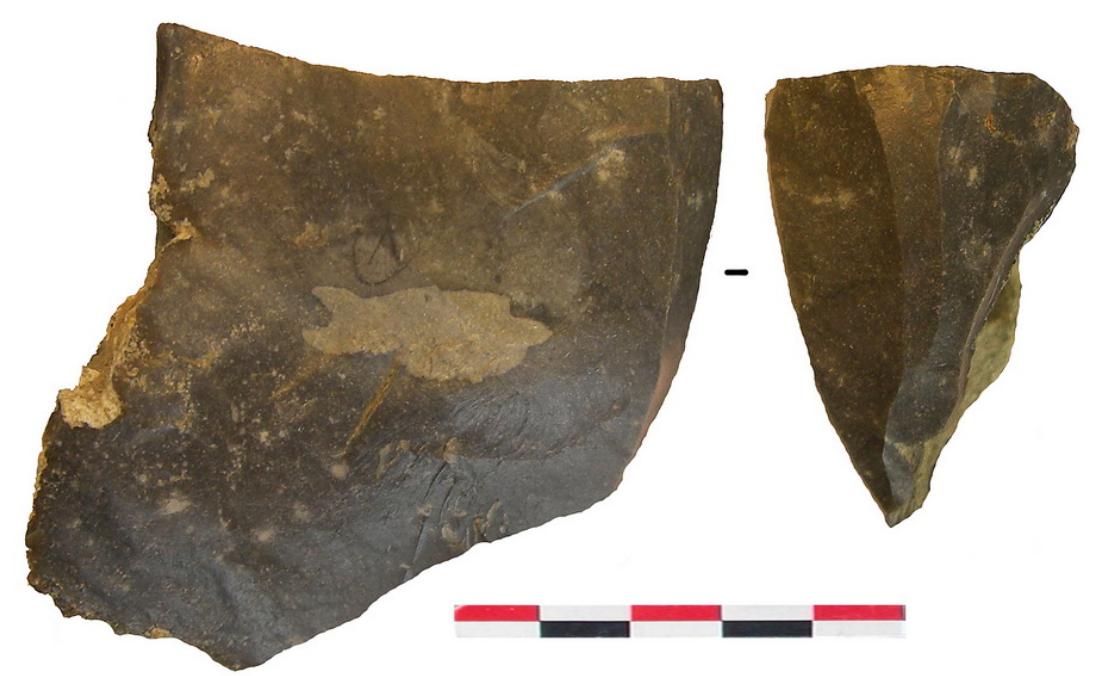

Figure 5. Oeudeghien (Belgique). Nucléus à lamelles sur tranche d'éclat (Crombé et al. 1992) (photo: P. Allard). (L'échelle est de $5 \mathrm{~cm}$ divisée en segments de $1 \mathrm{~cm}$.)

Figure 5. Oeudeghien (Belgium). Bladelet core on flake edge (Crombé et al., 1992) ( photo: P. Allard). (Scale bar is $5 \mathrm{~cm}$ wide, divided into $1 \mathrm{~cm}$ sections.)

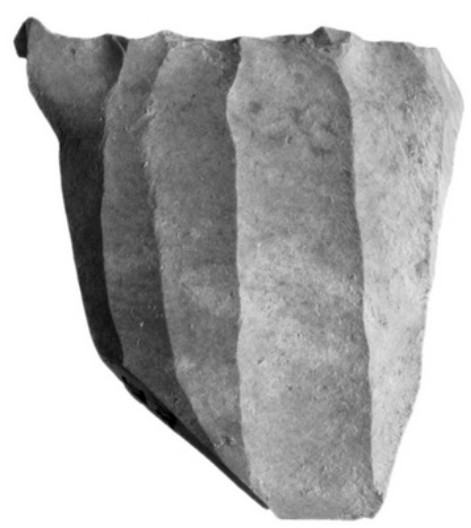

\section{$1 \mathrm{~cm}$}
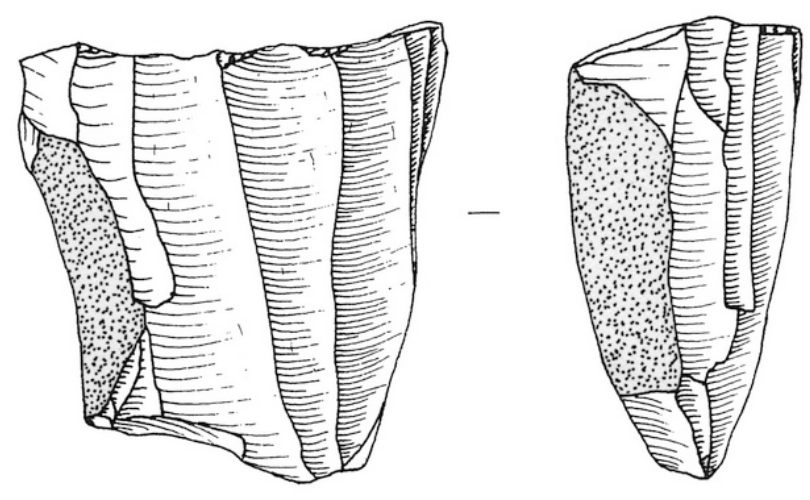

$(1 / 1)$
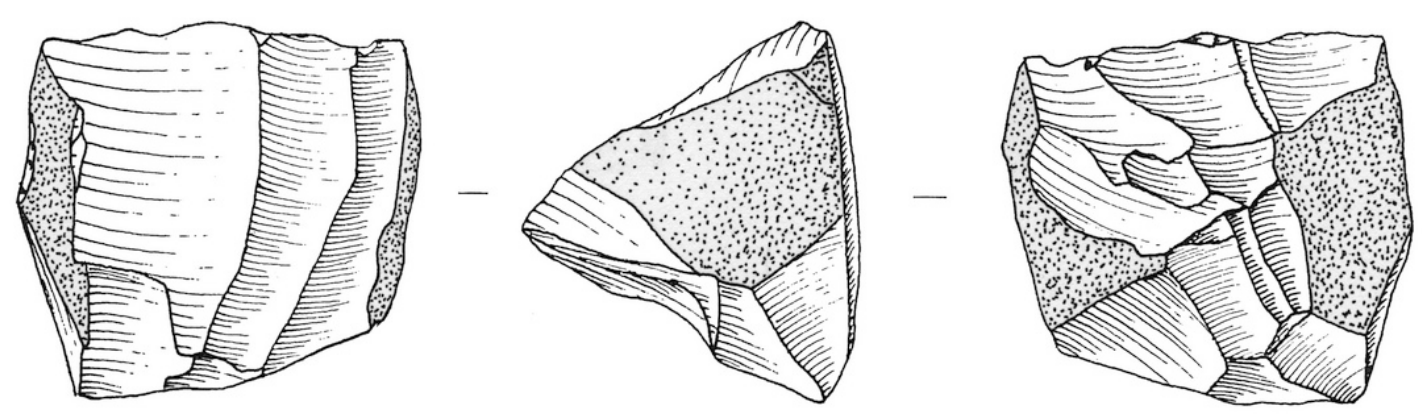

Figure 6. Ruffey-sur-Seille «A Daupharde ». Nucléus avec une surface lamellaire quadrangulaire (Seara et al. 2002: fig. 82 et 83 et Séara 2014).

Figure 6. Ruffey-sur-Seille «A Daupharde». Core with a quadrangular lamellar surface (Seara et al. 2002: figs. 82 and 83; Seara 2014).

On peut mentionner une variante dans la collection de «la Bouloie » en Haute-Marne (Amiot 1996), qui correspond à une collection de surface majoritairement dominée par les productions au punch et des trapèzes. On y observe un débitage lamino-lamellaire dominant sur des blocs orthogonaux, à débitage frontal et unipolaire mais avec des plans de frappe lisses très inclinés (Figure 7). Le détachement des produits laminaires présente un procédé de microfacettage du bord du plan de frappe au préalable qui oriente l'angle de chasse vers 
l'angle droit (Lautridou 2010). Dans ce cas, le microfacettage final permet une correction de l'angle de chasse vers l'angle de confort reconnu d'après les expérimentateurs pour la percussion indirecte.
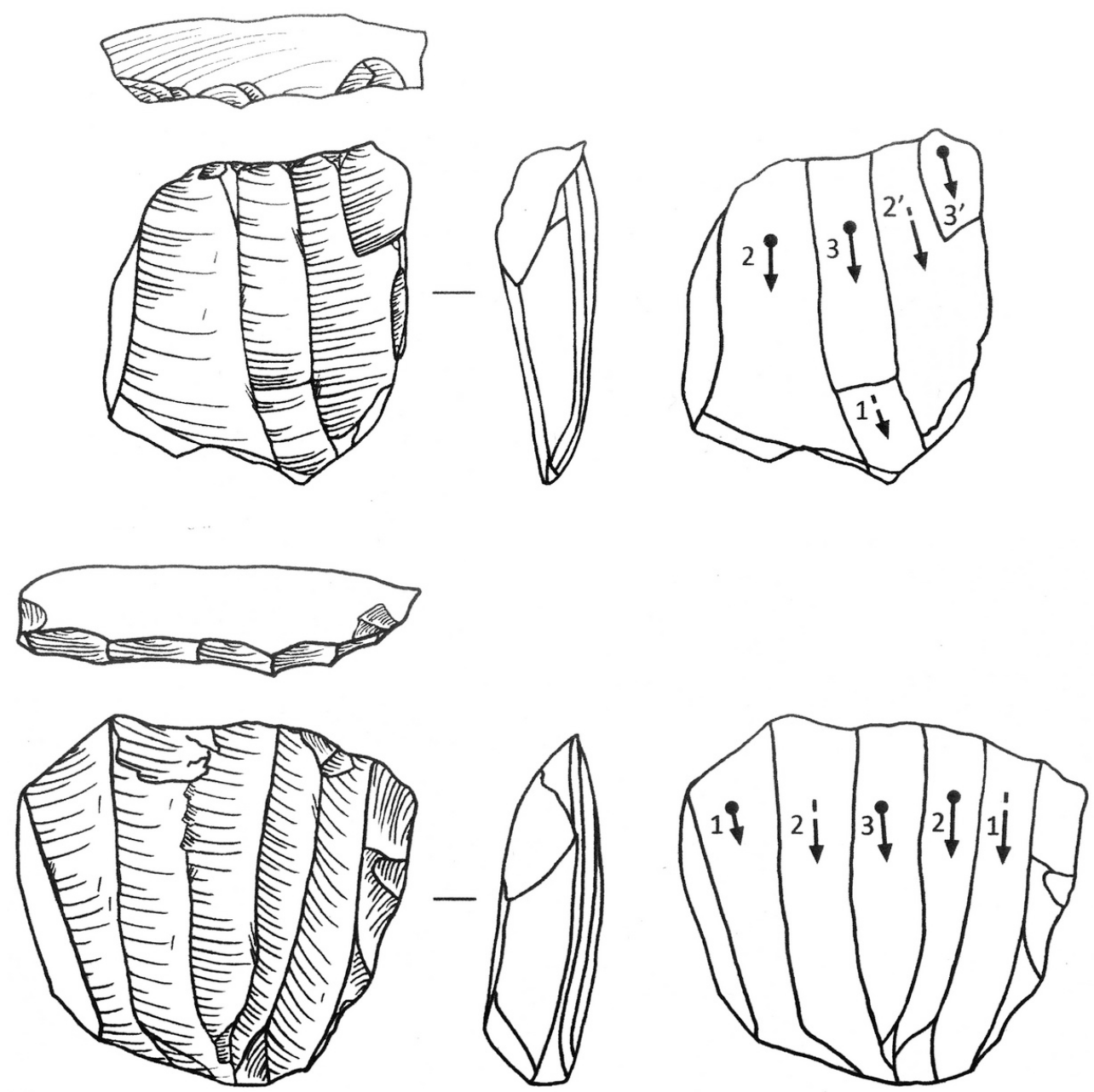

Figure 7. La Bouloie à Crenay (Haute-Marne). Nucléus à débitage frontal et unipolaire avec des plans de frappe lisses très inclinés (dessins: Lautridou 2010)

Figure 7. Core with frontal and unipolar debitage with flat inclined striking platform (drawings: Lautridou 2010)

Une seconde composante se discerne dans les études en cours du Tardenois qui met en lumière une production de supports de petits gabarits sur des plans très inclinés mais sans microfacettage avant le détachement des produits lamellaires. C'est le cas notamment à l'Allée Tortue ou encore à Mauregny en Haye (fouilles Hinout). Cette variante totalement inédite reste encore à bien documenter mais la régularité parfois extrême des produits permet d'avancer sans risque qu'il s'agit bien de la percussion indirecte. Les talons sont lisses et soigneusement abrasés mais avec un angle de chasse très fermé, parfois de l'ordre de $45 / 50^{\circ}$, ce qui a priori n'est pas dans les limites habituelles reconnues pour le débitage au punch.

Ces débitages avec des plans de frappe aménagés par facettage large et microfacettage du bord du plan ont été reconnus également dans l'Ouest de la France dans le Retzien (Marchand 1999). L'exploitation principale est réalisée sur des galets côtiers avec une mise en forme à la percussion dure. L'initialisation du débitage profite des convexités naturelles offertes par les galets. Les surfaces laminaires sont étroites car installées sur les parties minces des volumes. Le débitage est frontal avec une faible convexité latérale qui est entretenue par des lames latérales anguleuses de section triangulaire. 
Le plan de frappe est ravivé par des éclats et le bord est localement aménagé par un micro facettage qui se traduit par un très fort taux de talons facettés sur les produits lamellaires. Le débitage est unipolaire simple ou à séquences successives.

\section{Quelques éléments de réflexion sur la variabilité des débitages}

\subsection{Un concept commun}

De ces différentes chaînes opératoires, un standard semble désormais se dessiner :

- une préparation initiale faible, notamment avec l'emploi récurrent de surfaces et arêtes naturelles favorables pour l'initialisation du débitage. Néanmoins, les remontages montrent qu'une étape de mise en forme, parfois appelée dégrossissage peut être importante. Cependant, l'installation d'une crête antérieure apparaît comme un procédé peu exploité dans ces débitages.

- un débitage unipolaire « frontal » sur des nucléus à flancs orthogonaux sur surface laminaire quadrangulaire et peu cintrée. On pourrait remplacer le terme de frontal par un débitage quart tournant. Il est souvent mentionné que l'installation de la surface laminaire se fait sur les parties étroites des blocs mais c'est plutôt une règle dans les débitages laminolamellaires car cela garanti un contrôle plus simple des convexités latérales et distales et assure le meilleur volume pour la production.

- des supports réguliers et rectilignes, à 3 pans selon un rythme 212'. Les modules des produits comprennent une variation assez importante mais qu'il est difficile de quantifier précisément. Le terme de «lamino-lamellaire » est d'ailleurs fréquemment employé car il fait référence à une tradition ancienne de quantifier la limite entre lames et lamelles à 10 mm de large. Or, le module le plus fréquent oscille entre 8 et $12 \mathrm{~mm}$.

En accord avec G. Marchand, le débitage de l'Essart A tel qu'il est décrit (Marchand 2009) semble correspondre au dénominateur commun des modes de production à la percussion indirecte du second Mésolithique (Figure 8). Il est parfois qualifié de débitage frontal sur des nucléus à table resserrée (Marchand 2014a)

Néanmoins, une variabilité existe qui se manifeste sur la gestion du plan de frappe et les procédés préparatoires au détachement des produits lamellaires (plan de frappe facetté, parfois très incliné et les talons facettés des lamelles versus talons exclusivement lisses).

Il faut donc s'interroger sur les différents éléments qui peuvent contribuer à son interprétation.

\subsection{Réflexions sur la variabilité}

Un retour aux données des expérimentateurs et des critères descriptifs de la percussion indirecte permet de mettre en lumière les éléments de la variabilité.

Les travaux des expérimentateurs modernes de la taille du silex ont permis d'aborder l'identification des techniques de percussion. Les techniques peuvent être reconnues par l'examen attentif des nucléus (plan de frappe, micro-morphologie des contre-bulbes, géométrie de surface de taille, régularité des enlèvements) et des produits dont la partie proximale livre un grand nombre d'observation (préparation du point d'impact, angle de chasse et d'éclatement, etc.). Les expérimentations systématiques montrent toute la difficulté de reconnaître les techniques que seule l'étude d'une série nombreuse peut permettre un bon diagnostic. La mise en garde de J. Tixier (1982) nous rappelle à juste titre que cette discipline est l'apanage des expérimentateurs, l'évolution des connaissances dans cette démarche étant parallèle à l'expérience acquise de ces derniers au fur et à mesure de leurs travaux. Concernant les critères de diagnose pour la percussion indirecte, on peut se reporter aux nombreux travaux de Jacques Pelegrin à la fois concernant l'historique et les critères de diagnose 
(Bordes \& Crabtree 1969; Pelegrin 1988; 1991; 2006; 2012 ; Pelegrin \& Riche 1999). Avec les connaissances désormais acquises des expérimentateurs sur cette technique de percussion, on sait que le débitage au punch s'exprime efficacement avec des nucléus à plan de frappe lisse et un angle qualifié de confort entre 80 et $90^{\circ}$. L'abrasion des corniches peut être partielle ou absente puisque le punch permet d'assurer la précision de la zone d'impact. En fonction des séries lithiques du second Mésolithique que nous venons de décrire, il apparaît que la méthode de l'Essart A est conforme à ce qu'on pourrait attendre d'une série archéologique au punch et s'inscrit parfaitement dans les modalités de cet outil de percussion. Cela met en avant les différences avec les autres variantes qui a priori ne sont pas induites par l'utilisation du punch. On pense notamment au nucléus à plan très inclinés avec microfacettage du plan de frappe dans l'est de la France ou au Retzien en Bretagne. Ces différences sont donc des manifestations de manières de faire distinctes.
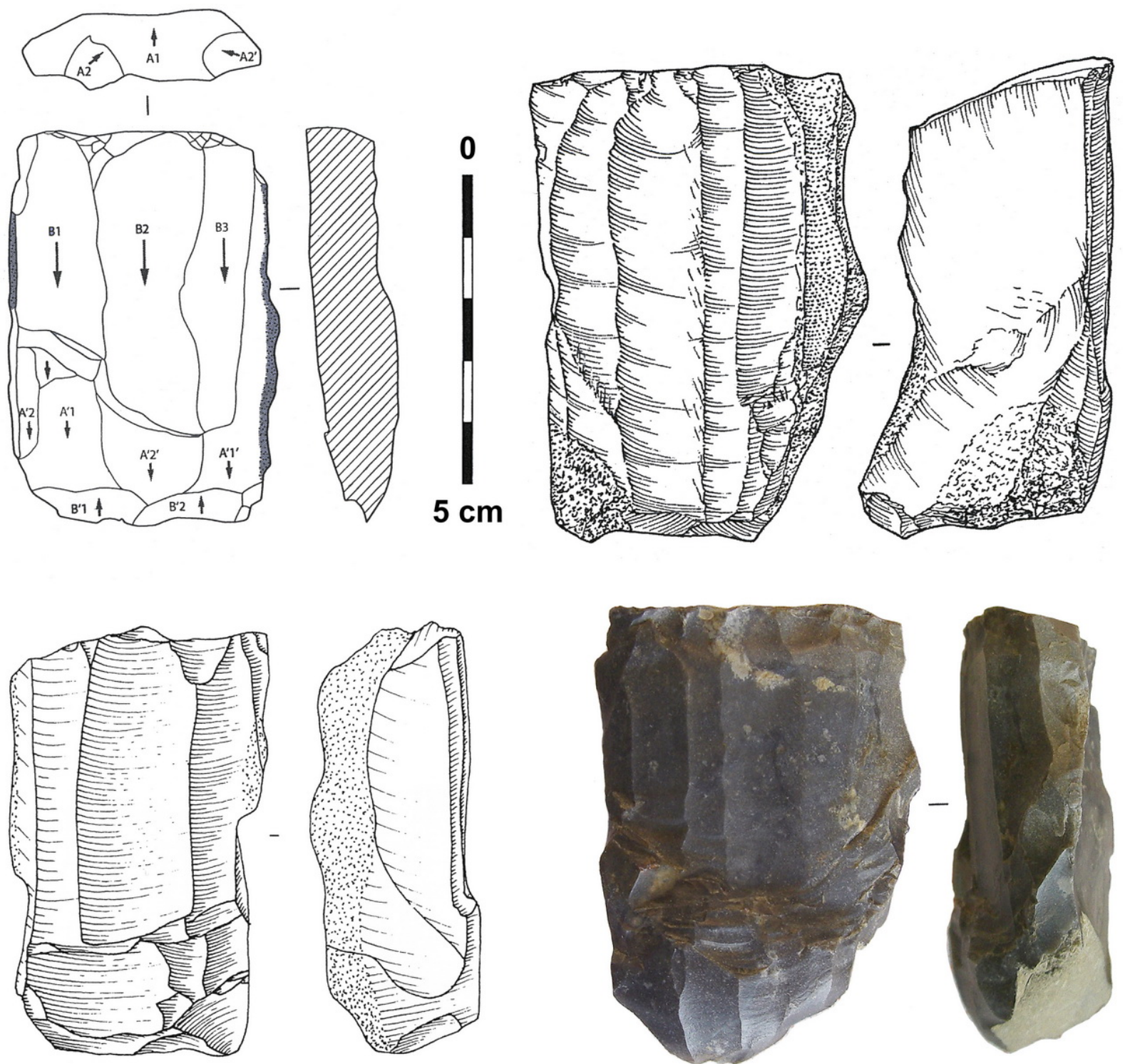

Figure 8. Nucléus à lamelles à table quadrangulaire et flancs orthogonaux des Essarts (Marchand 2009), Lhéry (Bostyn \& Seara 2011), Ruffey-sur-Seille « A Daupharde » (Seara et al. 2002) et Oeudeghien (Crombé et al. 1992) (photo: P. Allard).

Figure 8. Core with quadrangular bladelet surface and orthogonal flanks of the Essarts (Marchand 2009), Lhéry (Bostyn \& Seara 2011), Ruffey-sur-Seille “A Daupharde” (Seara et al. 2002) and Oeudeghien (Crombé et al. 1992) (photo: P. Allard). 
Une des caractéristiques de l'arrivée des industries à trapèzes en Méditerranée occidentale est l'apparition de la pression dans le courant castelnovien. Le débitage à la pression ne semble pas s'être développé en France septentrionale. Les travaux de Grégor Marchand dans l'ouest ne mentionnent pas cette technique de percussion sur toute la façade atlantique de même qu'elle n'est pas reconnue dans le secteur tardenoisien. On peut juste mentionner un nucléus, hors contexte précis, qui évoque un débitage à la pression à Choisyau-Bac «La Bouche d'Oise » (Gueret et al. 2009) (Figure 9), retrouvé dans un palimpseste d’occupations mésolithiques et néolithique. Sa structure générale évoque le Mésolithique récent mais cette pièce est bien isolée pour la région. Dans les séries de l'est, le problème est plus complexe. Si l'on peut suivre l'avis de F. Séara sur l'emploi de la percussion indirecte, notamment à Ruffey, il faut reconnaître que certains nucléus sont à la limite de nos connaissances expérimentales et la question de la pression doit être posée. Ainsi dans la série de la "Bouloie », les dimensions très réduites des nucléus épuisés posent le problème du maintien du bloc par percussion indirecte puisque le choc induit par ce mode de percussion induit que le nucléus doit encore faire une certaine masse pour ne pas trop vibrer sous l'impact. Or certaines pièces semblent bien en deçà de cette "masse critique ». Il reste donc encore un travail expérimental important à développer pour bien cerner les critères de diagnose entre la pression et la percussion indirecte pour les petits débitages lamellaires.

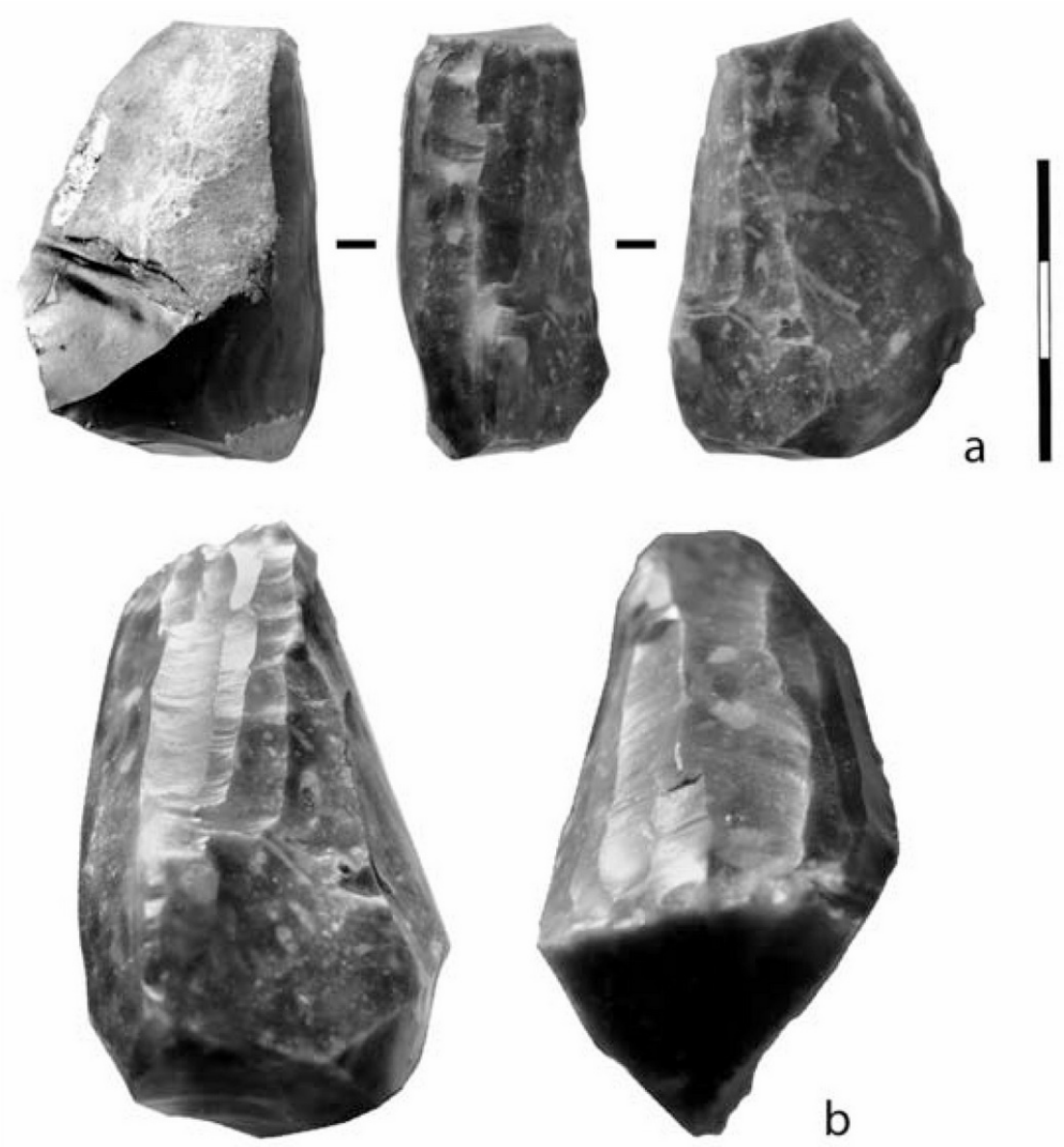

Figure 9. La “Bouche d’Oise”, nucléus à lamelles débité par pression (Gueret et al. 2009). (L’échelle est de 3 cm divisée en segments de $1 \mathrm{~cm}$.)

Figure 9. La "Bouche d'Oise", bladelet core with pressure technique (Gueret et al. 2009). (Scale bar is $3 \mathrm{~cm}$ wide, divided into $1 \mathrm{~cm}$ sections.)

Il faut remarquer que toutes ces variantes ou au contraire le débitage "standard » ne semblent pas contraint par la matière première. Par exemple à Ruffey, Lhéry ou à l'Essart, 
malgré une certaine diversité des matériaux exploités, les méthodes sont stables. L'emploi de blocs volumineux est bien attesté mais une opération préalable est alors mise en place pour fournir des volumes prêt à être débités, jouant notamment avec les diaclases ou les points de faiblesse de la matière première (Marchand 2009; Séara \& Bostyn 2009). Le débitage de gros éclats supports est également attesté mais semble réservé au nord de notre zone d'étude. L'étude d'Oedoughien est intéressante car la quantité de silex et la composition du mobilier indique clairement qu'il s'agit un site de production, pourtant a priori éloigné des gîtes de matières premières. On peut donc parfaitement envisager que ces éclats supports ont été transportés pour être débités à différents endroits, parfois éloignés de plusieurs dizaines de kilomètres des gîtes primaires.

Le débitage à la percussion indirecte comporte donc des variantes indépendantes de la matière première qui se manifestent dans les procédés de débitage comme l'abrasion des corniches ou le micro-facettage du plan de frappe avant le débitage, mais également dans la gestion des volumes à débiter.

\subsection{La difficile question de la chronologie}

La chronologie absolue fait cruellement défaut pour la compréhension des mécanismes d'apparition et d'évolution des industries à trapèzes dans notre zone d'étude en raison de l'ancienneté de nombreuses fouilles et des contextes sédimentaires défavorables (nombreux palimpsestes sur sols sableux acides). C’est pourtant un élément crucial puisque cela permettrait de pouvoir rendre compte de l'évolution du système technique de ces populations.

C'est la séquence de la vallée de la Somme qui apporte le plus d'informations. Les datations les plus anciennes des trapèzes ne sont pas directement associées au mobilier. Elles concernent quelques petits trapèzes latéralisés à gauche dans un niveau pris entre deux couches de tourbe datées entre 7800 et 7500 BP à la Chaussée-Tirancourt (Ducrocq 2001). Une datation sur charbon est également possible à Maurégny-en-Haye vers $7390 \pm 160 \mathrm{BP}$, toujours pour des petits trapèzes latéralisés à gauche. On ne dispose pas d'autres éléments, ni même réellement de séries que l'on pourrait attribuer avec certitude à l'apparition du phénomène des trapèzes pour le Bassin parisien ou le nord. En revanche, si on admet que la percussion indirecte est bien concomitante avec les trapèzes, il faut mentionner une découverte inédite qui nous provient de la vallée de l'Aisne. Une sépulture sur le site de Cuiry-lès-Chaudardes (Ilett 1998) livre un inhumé accompagné d'un collier en vertèbres de brochet et d'une série de 3 lamelles qui sont sans ambiguïté débitées à la percussion indirecte (Figure 10). La datation de cette sépulture est de 7400 BP \pm 60 BP, soit 6414-6099 cal BCE après calibration, ce qui fait de cet ensemble clos le plus ancien pour la datation de l'arrivée de la percussion indirecte dans le quart nord-est de l'Europe. On peut raisonnablement penser qu'elle est antérieure à 6200 cal BCE (Figure 11).

Dans l'Ouest, la reprise des fouilles et les nouvelles datations effectuées à Berg-er-Vil montre l'ancienneté de cette occupation qui reste le premier jalon de l'apparition des industries à trapèzes, vers 6200-6000 cal BCE (Marchand et al. 2016).

Le seul ensemble clos dans le nord ayant livré des trapèzes est la sépulture de Concevreux également dans l'Aisne (Robert et al. 2015) qui comportait une crémation avec un dépôt d'objets divers (andouillers de cerf, canines de sanglier et un objet en calcaire). Malheureusement les deux datations réalisées sur un os humain et sur un des objets du dépôt livre un écart très important (5470-5370 cal BCE et 6070-5980 cal BCE). Il faut noter que la sépulture pourrait être finalement plutôt ancienne et il semble raisonnable de la caler dans la première moitié du $6^{\mathrm{e}}$ millénaire. Le petit assemblage lithique comporte des lames et lamelles clairement identifiées à la percussion indirecte et une série de quatre trapèzes rectangles sans retouche inverse (Figure 10). 


\section{Concevreux}
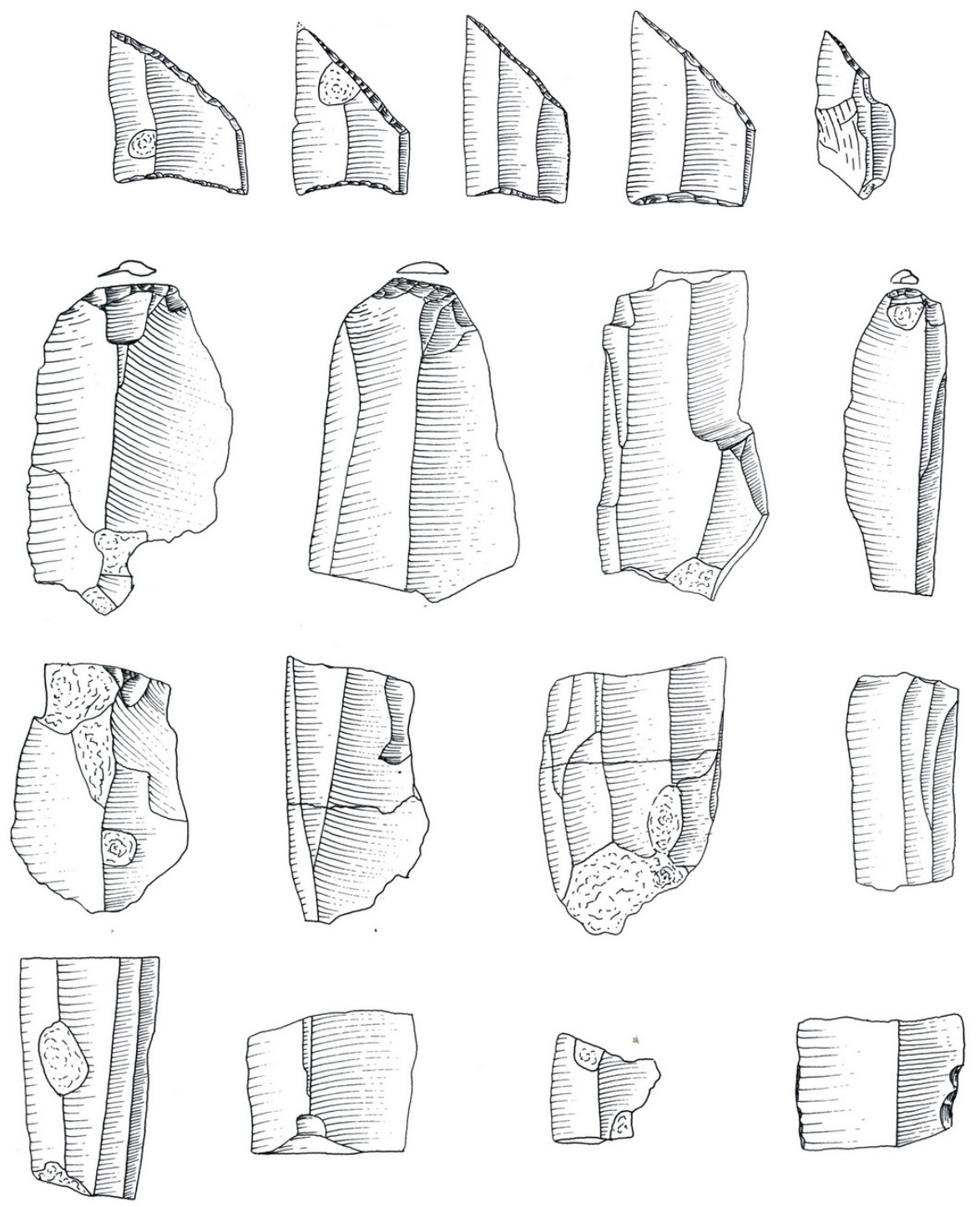

\section{Cuiry-lès-Chaudardes}
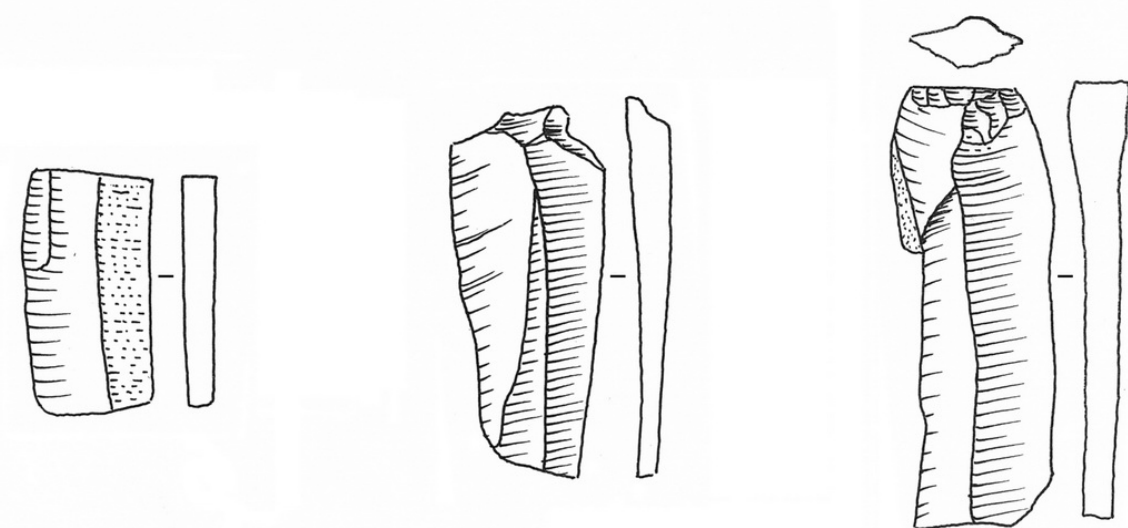

Figure 10. Mobilier lithique des sépultures mésolithiques de Concevreux (Robert et al. 2015) et de Cuiry-lèsChaudardes (Ilett 1998). Etudes et dessins P. Allard. (L'échelle est de $5 \mathrm{~cm}$ divisée en segments de $1 \mathrm{~cm}$.)

Figure 10. The lithic material of the Mesolithic graves of Concevreux (Robert et al., 2015) and Cuiry-lèsChaudardes (Ilett 1998). Studies and drawings P. Allard. (Scale bar is $5 \mathrm{~cm}$ wide, divided into $1 \mathrm{~cm}$ sections.) 


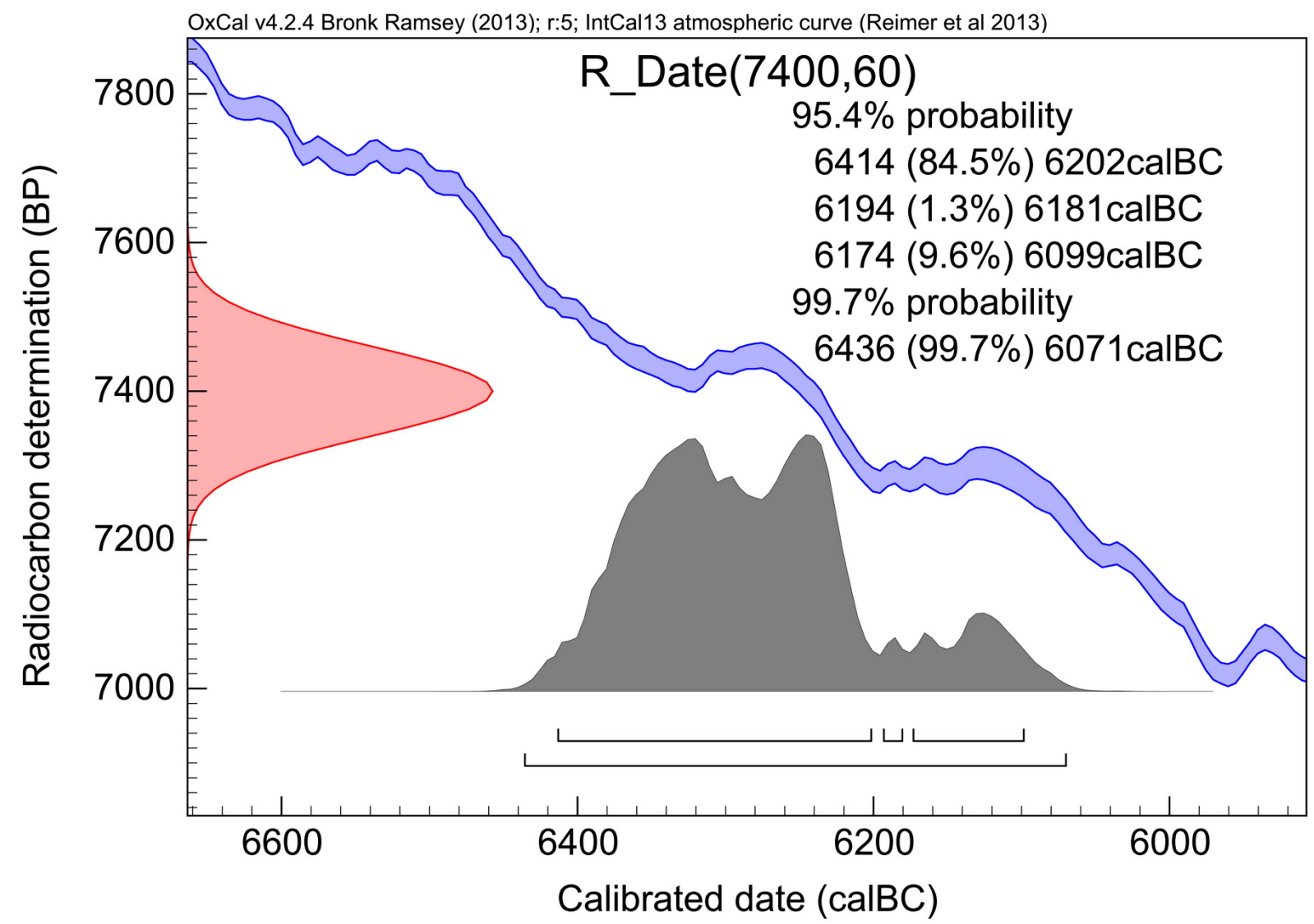

Figure 11. Date calibrée de la sépulture de Concevreux, selon OxCal v4.2.4 Bronk Ramsey (2013); r: 5; IntCal13 atmospheric curve (Reimer et al. 2013).

Figure 11. Calibrated date of the grave of Concevreux, according to OxCal v4.2.4 Bronk Ramsey (2013); r: 5; IntCal13 atmospheric curve (Reimer et al., 2013).

Les autres dates disponibles sont plus récentes et s'échelonnent dans le $7^{\mathrm{e}}$ millénaire. Le problème est qu'il est toujours délicat d'associer les ensembles ayant livré des informations détaillées sur les modes de débitage avec des datations précises. Ainsi, en reprenant les sites décrits dans la partie précédente, les Essarts n’ont pas livré de datations absolues et l'assemblage très varié d'armatures laisse supposer une occupation répétée sur une longue durée. C'est également le cas de Lhéry dont les datations disponibles couvrent pour la majorité une bonne partie du $6^{\text {e }}$ millénaire (6400-6200 cal BCE, 5480-5250 cal BCE, 53004940 cal BCE et 5290-4950 cal BCE, Seara \& Bostyn 2009). La datation du niveau R1 de Ruffey est plus centrée vers le milieu du $6^{\text {e }}$ millénaire à 5746-5479 cal BCE et 5576-5326 cal BCE (Seara et al. 2002). Elle est assez proche de l'ensemble bien daté de la Gilardière dans l'ouest (5667-5229 cal BC) tandis que le site de Beg-er-Dorchen semble plutôt occupé dans la première moitié du $6^{\mathrm{e}}$ millénaire à 5713-5344 cal BCE et 5701-5491 cal BCE (Marchand \& Perrin 2015).

Enfin, il faut mentionner une découverte très récente dont l'étude est en cours à Remilly dans les Ardennes (Souffi et al. 2015). Il s'agit de plusieurs occupations dont une est attribuée au Mésolithique final, bien datée entre 5630 et 5320 BCE (autour de 6500 BP) et qui livre des armatures évoluées à retouches inverses de type flèche de Belloy.

\section{La néolithisation, la fin des industries à trapèzes ?}

L’arrivée du Néolithique est globalement bien documentée pour la plupart des secteurs, mais on peut remarquer que les régions les mieux connues pour la fin du Mésolithique ne sont pas les mieux documentées pour l’arrivée du Néolithique (comme le Bassin de la Somme par 
exemple). A l'Est, le Rubané apparaît sur les marges du Rhin vers 5300 BCE et se propage vers le Bassin parisien, en Champagne vers 5200 s'étend jusqu'en Normandie à la fin du $6^{\mathrm{e}}$ millénaire (Demoule 2007). Dans l'Ouest, la situation est plus complexe avec le Néolithique ancien Atlantique dont on manque encore de précision sur les datations et en Bretagne, on connaît désormais des sites du groupe de BQ/Villeneuve Saint Germain dans le Finistère au début du $6^{\mathrm{e}}$ millénaire (Juhel 2014).

En ce qui concerne l'industrie lithique, différents éléments ont été avancés comme des témoins de l'implication des populations autochtones dans la néolithisation. Il apparaît à la fin de la séquence de la Céramique Linéaire qu'une grande zone qui s'étend du nord du Bassin parisien au Limbourg hollandais présente des similitudes fortes avec les armatures du Mésolithique terminal. En revanche, un contraste important existe avec les armatures des sites rubanés de l'est du Bassin parisien et de l'Alsace (absence de pointe asymétrique) où l'on ne trouve aucun point commun avec les séries mésolithiques. En tenant compte de la seule valeur identitaire des pointes de flèche, cela pourrait suggérer la possibilité d'une coexistence d'un modèle dual de néolithisation pour le Bassin parisien (Allard 2005). Nous avions sur ce sujet mis en lumière les différents éléments qui montrent des distinctions fortes entre le Rubané et ce que l’on connaît des armatures évoluées. Ainsi, certaines formes typologiques disparaissent et d'autres apparaissent au Rubané tandis que la latéralisation change également (Allard 2007; Allard \& Denis 2013). Les travaux menés depuis n’ont pas modifié cette vision en ce qui concerne les armatures. Ainsi, si une part des armatures du Néolithique ancien est bien proche de celle du mésolithique final, l'adéquation n'est que partielle et l'on doit tenir compte de la profondeur géographique et chronologique du courant rubané pour pouvoir bien comprendre la constitution de cette industrie. On peut également mentionner différents travaux qui évoquaient la possibilité d'emprunt par les mésolithiques d'une partie des armatures rubanées, notamment en ce qui concerne les flèches de Belloy (Crombé 2008, Ghesquière \& Marchand 2010). A ce titre, la découverte de l'assemblage à flèche de Belloy de Remilly (Souffi et al. 2015) démontre bien l'ancienneté de ce type qui est donc bien clairement antérieur au Néolithique ancien.

Le débitage principal et ubiquiste du Rubané est laminaire par percussion indirecte. Les produits laminaires sont assez courts (8-10 cm pour 1,5 à $2 \mathrm{~cm}$ de large en moyenne) rectilignes avec des bords parallèles, indifféremment à 2 ou 3 pans (Allard 2005).

Dans les séries étudiées, des différences sont perceptibles dans la conduite du débitage entre les séries omaliennes (Rubané de Hesbaye en Belgique) et celles du Rubané de l'Aisne. Le fait important est que le débitage laminaire est très homogène pour la France septentrionale et montre un débitage trois-quart tournant à tournant sur des nucléus unipolaire pyramidaux avec un facettage centripète centimétrique assez systématique du plan de frappe avec des talons lisses ou pseudo dièdre des produits laminaires (Figures 12 et 13). La mise en forme comprend une étape d'épannelage à la percussion dure puis de finalisation d'une crête antérieure ou de plusieurs crêtes à la percussion indirecte. L'angle de chasse est proche de l'angle droit et la préparation des lames comprend une abrasion de la corniche assez systématique. La découverte des nouveaux sites rubanés à l'Ouest, notamment Colombelles en Normandie, a confirmé l'homogénéité du système technique du Rubané (Billard et al. 2014). Ce type de débitage très caractéristique du Rubané se retrouve sous une forme similaire dans le groupe de Blicquy/Villeneuve-Saint-Germain qui lui succède (Bostyn 1994; Allard \& Bostyn 2006). Il faut noter que la production d'éclats apparaît sporadiquement sur des matériaux locaux, à l'exception des sites de la confluence Seine-Yonne où elle est majoritaire dès le Rubané (Augereau 2004). 


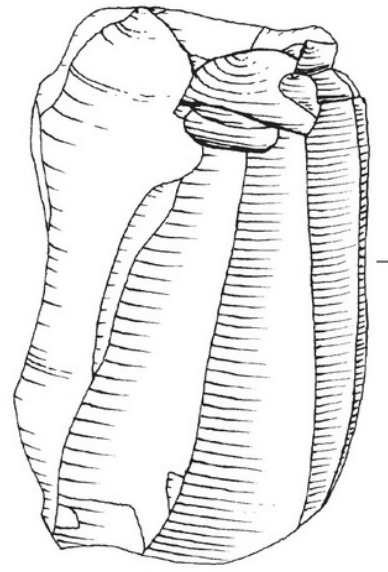

CCF nucléus 6 , st. 33, sénonien

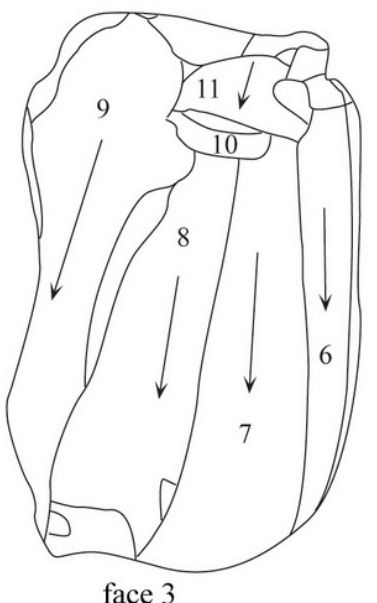

face 3
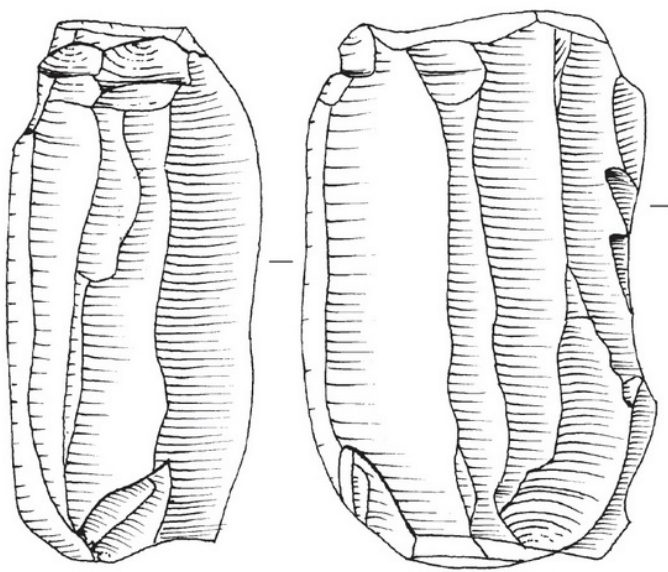

pas d'origine sur cette table
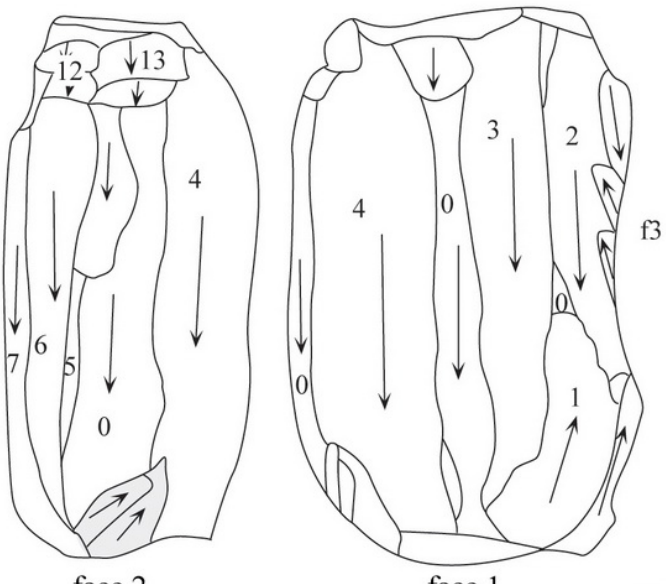

gauche

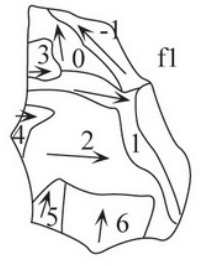

f2

plan de frappe
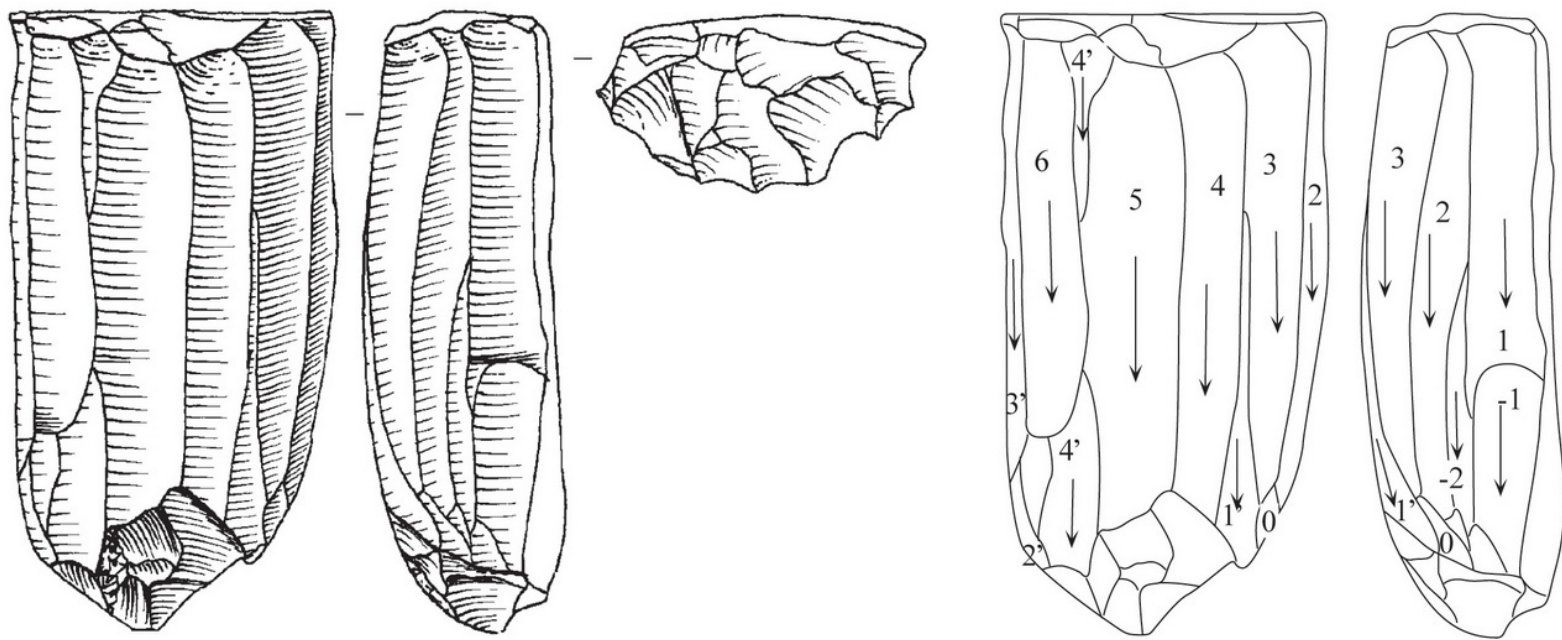

BVT nucléus 18 , st. 630 , tertiaire

Figure 12. Nucléus du Néolithique ancien (Rubané récent du Bassin de la Seine) de Cuiry-lès-Chaudares « les Fontinettes » et de Berry-au-Bac « Le vieux Tordoir » (Allard 2005). (L'échelle est de $5 \mathrm{~cm}$ divisée en segments de $1 \mathrm{~cm}$.)

Figure 12. Blade core of the Early Neolithic (Linearbandceramic of the Seine Basin) of Cuiry-lès-Chaudares "Les Fontinettes" and Berry-au-Bac "The Vieux Tordoir" (Allard 2005). (Scale bars are 5 cm wide, divided into $1 \mathrm{~cm}$ sections.) 


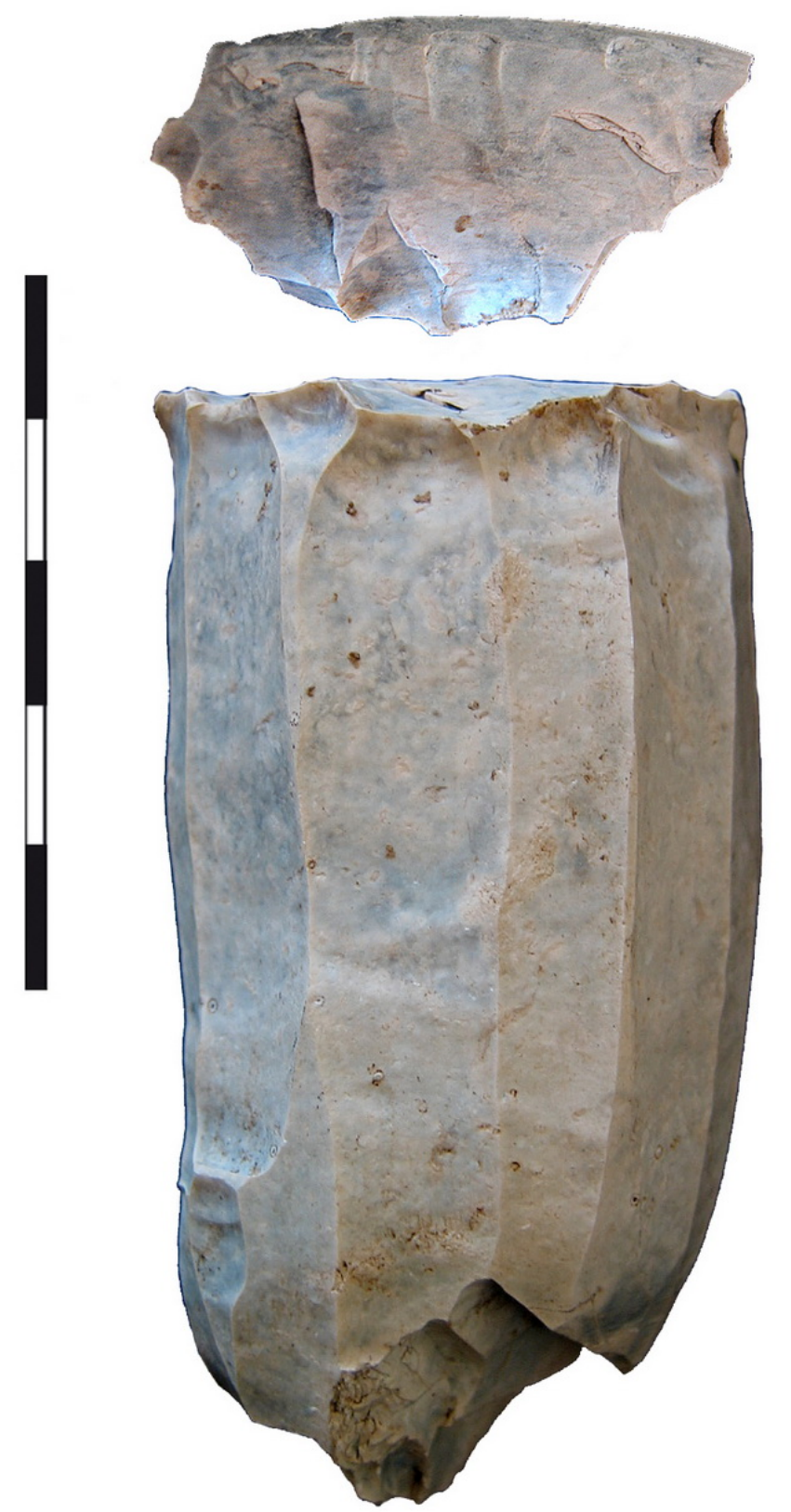

Figure 13. Nucléus du Néolithique ancien (Rubané récent du Bassin de la Seine) de Berry-au-Bac «Le vieux Tordoir » (Allard 2014). (L’échelle est de $5 \mathrm{~cm}$ divisée en segments de $1 \mathrm{~cm}$.)

Figure 13. Blade core of the Early Neolithic (Linearbandceramic of the Seine Basin) Berry-au-Bac "The Vieux Tordoir" (Allard 2014). (Scale bar is $5 \mathrm{~cm}$ wide, divided into $1 \mathrm{~cm}$ sections.)

Ainsi, si l'on peut considérer que l'arrivée du Néolithique ancien ne marque pas la disparition des trapèzes, on remarque une rupture franche en comparaison avec les grands sites mésolithiques de plein air. Les méthodes de débitages des productions lamellaires mésolithiques ne trouvent pas d'adéquation avec les sites du Néolithique ancien. Les méthodes de l'Essart A ou celle des sites de l'est de la France à micro-facettage et plan de frappe très inclinés du mésolithique sont distinctes des nucléus pyramidaux semi tournant à tournant, à facettage large du plan de frappe et talons lisses du Rubané ou du BQ/VSG (il n'y aucune lame à talon facetté dans le Néolithique ancien danubien). Il faut signaler également que le Néolithique ancien marque l'absence des lames Montbani mais il faut reconnaître que les études tracéologiques sur ce point sont rares. Il serait souhaitable de les développer afin 
d'évaluer précisément si ces lames à coches subsistent ou non dans les assemblages du Néolithique ancien danubien.

\section{Quelques éléments de synthèse en guise de conclusion}

Ainsi dans le complexe des industries à lames régulières et à trapèzes du $\mathrm{VII}^{\mathrm{e}}$ et $\mathrm{VI}^{\mathrm{e}}$ millénaire, coexistent différentes techniques et variantes du débitage des lames et lamelles régulières.

L'hypothèse actuelle voit une origine méridionale en tant que voie principale pour la diffusion de ces nouvelles armatures (Perrin et al. 2010; Marchand \& Perrin 2015). Le courant castelnovien peut être perçu comme une sorte de package technique associant techniques de débitage à la pression et à la percussion indirecte, des trapèzes et des lames encochées. Néanmoins, on ne peut totalement exclure une autre voie orientale, les lacunes documentaires et des datations dans le centre de l'Europe ne permettent pas de répondre avec certitude.

L'archéologie préventive cependant permet d'avancer sur ce problème car la multiplication des découvertes avec des ensembles clos ou des occupations moins mélangées apportent des nouveaux jalons qui offrent un meilleur cadre chronologique. Ainsi, l'arrivée de la percussion indirecte dans le Bassin parisien peut être désormais datée de 6200 BCE par la sépulture 711 de Cuiry-lès-Chaudardes, ce qui est nettement plus ancien que ce que l'on connaissait dans cette région (Perrin et al. 2010) et semble confirmer la datation indirecte des trapèzes de la Chaussée-Tirancourt (Ducrocq 2001; 2009). L’ambiguïté demeure sur la coexistence de trapèzes et d'armatures anciennes dans une étape première (Rozoy 1978). Néanmoins, au vu de ce que l'on connaît désormais sur la façade atlantique et en méditerranée (Binder et al. 2012; Marchand et al. 2016; Marchand \& Perrin 2015), il semble peu probable que ce fut le cas. En effet, les qualités intrinsèques des productions à la pression ou à la percussion indirecte (meilleure précision et contrôle du point d'impact, meilleure régularité et productivité) font douter de la coexistence des deux styles de débitages dont les armatures sont adaptées.

Malgré un concept commun, l'étude de ce «complexe » des industries à trapèzes montre une certaine variabilité du point de vue des méthodes de taille. Il semble se dégager des entités parfois bien circonscrites au sein de cet ensemble. C'est le cas du Retzien qui s'oppose nettement au Téviécien de par les procédés de préparation systématique par micro-facettage mais également par une excellente unité stylistique des types de trapèzes. Le débitage sur tranche d'éclat pour l'installation des surfaces laminaires semble pour le moment cantonné d'une zone allant du Bassin parisien à la Belgique. L'est de la France se démarque par des productions avec des talons facettés et des plans très inclinés. Il faudra donc donner du sens à ces différentes pratiques qui pour le moment sont des résultats bruts.

Enfin, il reste encore à mieux préciser les assemblages typologiques. Nous ne disposons pas encore suffisamment de séries qui permettent de bien comprendre l'évolution des différents types de trapèzes et triangles dérivés et si certaines méthodes de débitages sont particulières à certains types d'armatures. Malgré les imprécisions des datations et la difficulté de démêler les assemblages d'armatures, on peut retenir qu'il existe probablement des moments où certains types dominent. C'est le cas par exemple des trapèzes symétriques à Beg-er-Vil ou encore des trapèzes et triangles asymétriques avec retouches inverses de la fin du Mésolithique. Les études technologiques, notamment sur les supports, aideront surement à clarifier certaines productions particulières. Par exemple, l'apparition des trapèzes à bases décalées et des rhombes dans le Bassin parisien montre un moment où la production change, notamment par le débitage de supports nettement plus larges qui pourraient traduire une modification du concept habituel des nucléus à table quadrangulaire et flanc orthogonaux. Ce 
travail reste totalement à faire et il est tributaire d'une meilleure résolution chronologique et des contextes des séries du Second Mésolithique.

\section{Références}

Allard, P. 2005, L'industrie lithique des populations rubanées du Nord-Est de la France et de la Belgique. Verlag Marie, Leidorf, Rahden, 242 p. (in French) ("The lithic industry of Bandkeramik populations in north-east France and Belgium”)

Allard, P., 2007, The Mesolithic-Neolithic transition in the Paris Basin: a review. In: Going Over (Whittle A., \& Cummings, V., Eds.), Proceedings of the British Academy Vol. 144, Oxford University Press, Oxford: p. 213-224. (en anglais) ("La transition Mésolithique-Néolithique dans le Bassin parisien : aperçu général”) doi:10.5871/bacad/9780197264140.003.0011

Allard, P., \& Bostyn, F. 2006, Genèse et évolution des industries lithiques danubiennes du Bassin parisien, In: Contribution des matériaux lithiques dans la chronologie du Néolithique ancien et moyen en France et dans les régions limitrophes (Allard, P., Bostyn, F. \& Zimmermann, A., Eds.), BAR International Series Vol. 1494, Archaeopress, Oxford: p. 28-52. (in French) ("Genesis and evolution of the Danubian lithic industries of Paris Basin”)

Allard, P., \& Denis, S. 2013, Origine et fin des traditions danubiennes : l'industrie lithique du Néolithique ancien de la vallée de l’Aisne. In chapter: Autour du Néolithique ancien. Les outils du changement : critique des méthodes (Session H) (Perrin, T., Manen, C., Marchand, G., Allard, P., Binder, D., \& Ilett, M., Chapter Eds.), In: Transitions, ruptures et continuité durant la Préhistoire, Actes du 27e Congrès préhistorique de France (Bordeaux - Les Eyzies, 2010) (Jaubert, J., Fourment, N., \& Depaepe, P., Eds.), Société préhistorique française, Paris: p. 465-483. (in French) ("Origin and ending of the Danubian traditions: the lithic industry of the Early Neolithic period of the Aisne valley")

Allard, P., Marchand, G., Perrin, T., Binder, D., Crombé, P., Garcia Puchol, O., Michèle, S., Valdeyron, N. sous presse, The Late Mesolithic of Western Europe: a technological approach of the blade and trapeze industries. In: Méso'2010. Proceedings of the Eight International Conference on the Mesolithic in Europe, Santander (ES), sept. 2010 (Arias, P., Ed.), Oxbow Books, Oxford: 15 p. (en anglais) (“Le Mésolithique récent en Europe occidentale : approche technologique de l'industrie des lames et trapèzes”)

Amiot, C. 1996, Les industries préhistoriques du site de La Bouloie à Crenay (Haute-Marne) : Première partie : l'Epipaléolithique et le Mésolithique. Préhistoire et Protohistoire en Champagne Ardenne, 20: 15-33. (in French) ("The prehistoric industries from La Bouloie site at Crenay, Haute-Marne”)

Augereau, A. 2004, L'industrie du silex du Ve au IVe millénaire avant J.-C. dans le sud-est du Bassin parisien. Documents d’Archéologie Française Vol. 97, Éditions de la Maison des Sciences de l'Homme, Paris, 220 p. (in French) ("The industry of the flint from the 5 th to the 4th millennium BC in the southeast of Paris Basin”)

Biagi, P. \& Starnini, E. 2016 - The Origin and Spread of the Late Mesolithic Blade and Trapeze Industries in Europe: Reconsidering J. G. D. Clark’s Hypothesis Fifty Years After. In: Interactions, changes and meanings. Essays in honour of Igor Manzura on the occasion of his 60th birthday (Țerna, S. \& Govedarica, B., Eds), Kishinev 2016, 
Stratum publishing house, Kishinev: p. 20-33. (en anglais) (“L'origine et la diffusion de l'industrie des lames et trapèzes récente en Europe”)

URL: https://www.eanthropology.com/English/Catalog/Archaeology/STM_DWL_lJ2q_ c9EZ4gYLTdiC.aspx

Billard, C., Bostyn, F., Hamon C., Meunier K. (Eds.) 2014, L'habitat du Néolithique ancien de Colombelles «Le Lazzaro » (Calvados). Mémoires de la Société Préhistorique Française, Tome 58, Société Préhistorique Française (SPF), Paris, 408 p. (in French) ("The early neolithic settlement of Colombelles « Le Lazzaro » (Calvados")

Binder, D. 1987, Le Néolithique ancien provençal, typologie et technologie des outillages lithiques, Gallia-Préhistoire, 24e supplément, Éditions du CNRS, Paris 205 p. (in French) ("The early neolithic provençal, typology and technology of lithic tools")

Binder, D., Collina, C., Guilbert, R., Perrin, T., Garcia Puchol, O. 2012, Pressure knapping blade production in the North-Western Mediterranean Region during the 7th millennium cal B.C. P.M. In: The Emergence of Pressure Blade Making: from Origin to Modern Experimentation (Desrosiers, P., Ed.), Springer Verlag, Heidelberg: p. 199-218. (in English) ("Les productions laminaires à la pression dans la région de la méditerranée nord occidentale au $7^{\mathrm{e}}$ millénaire avant notre ère”) doi:10.1007/978-1-4614-2003-3_7

Bordes, F. \& Crabtree, D. 1969, The Corbiac blade technique and other experiments. Tebiwa, 12(2): 1-21. (in English) ("La technique laminaire de Corbiac et autres expérimentations”)

Bostyn, F. 1994, Caractérisation des productions et de la diffusion des industries lithiques du groupe néolithique du Villeneuve-Saint-Germain, Unpublished PhD thesis, University of Paris X Nanterre, Paris, 744 p. (in French) ("Characterization of the productions and diffusion of the lithic industry of the Villeneuve-Saint-Germain group”)

Bostyn, F. \& Seara, F. (Eds.), 2011, Occupations de plein-air Mésolithique et Néolithique : le site de La Presle à Lhéry (Marne). Société Préhistorique Française, Travaux n 10 , Société préhistorique française, Paris, 289 p. (in French) ("Mesolithic and Neolithic outdoor occupations: The site of Lhery « la Presle (Marne)")

Clark, J. G. D. 1958, Blade and Trapeze Industries of The European Stone age. Proceedings of the Prehistoric Society, 24(2): 24-42. (in English) ("Industrie des lames et trapèzes de l’Age de la Pierre en Europe”) doi:10.1017/S0079497X00016716

Crombé, Ph. 2008, Contacts et échanges entre chasseurs-cueilleurs et agriculteurs durant le 6ème et 5ème millénaire avant J.C. dans l'ouest de la Belgique. In: Fin des traditions danubiennes dans le Néolithique du Bassin parisien et de la Belgique (5100-4700 BC) ; autour des recherches de Claude Constantin (Burnez-Lanotte L., Ilett M. \& Allard P., Eds.), Mémoires de la Société Préhistorique Française Vol. 44, Société préhistorique française, Paris: p. 59-74. (in French) ("Contact and trade between hunter-gatherer and forager during the 6 th and 5 th millenium BCE in Belgium")

Crombé, P., Langohr, R., Velghe, M. 1992, Un site Mésolithique récent à Oeudeghien (Hainaut occidental). Notae Praehistoricae, 11(1991): 37-40. (in French) (“A late Mesolithic site at Oeudeghien (Hainaut occidental)”)

Demoule, J.P. (Ed.), Cottiaux, R., Dubouloz, J., Giligny, F., Jallot, L., Manolakakis, L., Marchand, G., Sénépart, I. 2007, La révolution néolithique en France, Editions la Découverte, Paris, 180 p. (in French) (“The Neolithic Revolution in France”) 
Deschamps, S. 2000, Apports de l'étude techno-économique de l'industrie lithique du Mésolithique récent de Ruffey-Sur-Seille «À Daupharde » (Jura), à la connaissance du Mésolithique. Mémoire de maîtrise, Université Paris 1, 114 p. (in French) ("The contribution of the techno-economic study of the lithic industry of the late Mesolithic site of Ruffey-Sur-Seille « À Daupharde » (Jura) to the knowledge of the Mesolithic”)

Ducrocq, T. 2001, Le Mésolithique du Bassin de la Somme. Insertion dans un cadre morphostratigraphique, environnemental et culturel, Lille, Publications du CERP Vol. 7, Centre d'études et de recherches préhistoriques (CERP), Lille, 253 p. (in French) ("The Mesolithic of the Somme basin. Morphostratigraphy, environemental and cultural framework")

Ghesquière, E., 2012, Le Mésolithique en Basse-Normandie. Thèse de doctorat, EHESS, Université Toulouse 2- Le Mirail, Toulouse, 2 Vol., 848 p. (in French) ("The Mesolithic of Low Normandy ")

Ghesquière, E. \& Marchand, G., 2010, Le Mésolithique en France, Archéologie des derniers chasseurs-cueilleurs. Editions La Découverte, Paris, 177 p. (in French) (“The Mesolithic in France, Archaeology of the last hunter-gatherers”)

Guéret, C., Pelegrin, J., Valentin, B. 2009, Révision taphonomiques à propos du Mésolithique moyen et récent à la « Bouche d'Oise » à Choisy-au-Bac (Oise). In: Paléolithique final et Mésolithique dans le Bassin parisien et ses marges (Valentin, B., Ed.), CNRS-UMR 7041, Projet collectif de recherche. Rapport d'activités pour 2009, Équipe d'Ethnologie préhistorique, Nanterre Cedex: p. 263-274. (in French) ("Taphonomic review of the middle and late Mesolithic of «Bouche d'Oise » at Choisy-au-bac (Oise)”) URL: https://halshs.archives-ouvertes.fr/hal-01381372/document

Hinout, J. 1989, Le gisement tardenoisien final du "Bois de Chinchy" commune de Villeneuve-sur-Fère (Aisne), Revue Archéologique de Picardie, 3(1): 15-26. (in French) ("The late tardenoisian site of "Bois de Chinchy" commune de Villeneuve-sur-Fère (Aisn)”) doi:10.3406/pica.1989.1550

Hinout, J. 1990, Le Tardenoisien Final III. Le gisement de la Baillette à Oulchy-la-Ville (Aisne). Bulletin de la Société préhistorique française, 87(8): 241-249. (in French) ("The late tardenoisian III. The site of Baillette à Oulchy-la-Ville (Aisn)") doi:10.3406/bspf.1990.9443

Ilett, M. 1998, Cuiry-les-Chaudardes «Les Fontinettes ». In: Bilan scientifique de la région Picardie 1998. Direction régionale des affaires culturelles de Picardie, Service régional de l'archéologie, Amiens: p. 26-27. (in French) (“Cuiry-les-Chaudardes « Les Fontinettes » (Aisn)”)

URL: http://www.culturecommunication.gouv.fr/content/download/26928/225242/versi on/2/file/BSR1998.pdf

Juhel, L. 2014, Un hameau du Néolithique ancien à Lannion «Kervouric» (Côtes-d’Armor): brève information. Internéo, (Actes de la Journée d'information du 22 novembre 2014, Paris) 10: 153-156. (in French) (“An early Neolithic hamlet at Lannion «Kervouric» (Côtes-d'Armor)» (Aisn)”) URL: http://www.prehistoire.org/offres/file_inline_src/515/515_pj_131216_181341.pdf

Lautridou, C. 2010, Etude d’une industrie du Mésolithique : «la Bouloie » (Haute-Marne), Mémoire de Master, Université de Paris Ouest Nanterre La Défense, Nanterre, 45 p. (in French) (“Study of a Mesolithic industry: « la Bouloie » (Haute-Marne)”) 
Ketterer, I. 1997, Les techniques et l'économie du débitage mésolithique d'Hangest « gravière II» Nord (Somme). In: Le Tardiglaciaire en Europe du nord-ouest (Fagnart, J.-P., \& Thévenin, A., Eds.), Actes du $119^{\mathrm{e}}$ Congrès National des sociétés historiques et scientifiques (Amiens, 26-30 octobre 1994), Édition du CTHS, Paris: p. 123-137. (in French) ("The techniques and the economy of the mesolithic débitage of d'Hangest « gravière II» Nord (Somme)”)

Kozlowski, S. K. 2009, Thinking Mesolithic. Oxbow Books, Oxford, 545 p. (in English) ("Penser Mésolithique")

Marchand, G. 1999, La néolithisation de l'ouest de la France : caractérisation des industries lithiques. BAR International Series Vol. 748, Archaeopress, Oxford, 487 p. (in French) ("The neolithisation of western France : caracterization of lithic industries")

Marchand, G. (Ed.), 2009, Des feux dans la vallée. Les habitats du Mésolithique et du Néolithique récent de l'Essart à Poitiers. Collection " Archéologie et Culture », Presses Universitaires de Rennes, Rennes, 246 p. (in French) ("Fire in the valley. The Mesolithic and Late Neolithic settlements of l'Essart at Poitiers")

Marchand, G. 2014a, Premier et second Mésolithique : Et au-delà des techniques ? In: Des techniques aux territoires : nouveaux regards sur les cultures mésolithiques. Actes de la table-ronde, 22-23 novembre 2012 (Henry, A., Marquebielle, B., Chesnaux, L., \& Michel, S., Eds.), P@lethnology, Vol. 6, Maison de la recherche, Toulouse: p. 9-22. (in French) (“First and Second Mesolithic: And beyond the techniques?”)

URL: http://blogs.univ-tlse2.fr/palethnologie/2014-revue/

Marchand, G. 2014b, Préhistoire atlantique. Fonctionnement et évolution des sociétés du Paléolithique au Néolithique. Editions Errance, Arles, 528 p. (in French) (“Atlantic prehistory. Functioning and evolution of the Paleolithic and Neolithic societies”)

Marchand, G., Dupont, C., Delhon, C., Desse-Berset, N., Gruet, Y., Laforge, M., Le Bannier, J-C., Netter, C., Nukushina, D., Onfray, M., Querré, G., Quesnel, L., Stéphan, P., Tresset, A. 2016, Retour à Beg-er-Vil: Nouvelles approches des chasseurs-cueilleurs maritimes de France Atlantique. In: Archéologie des chasseurs-cueilleurs maritimes. De la fonction des habitats à l'organisation de l'espace littoral (Dupont, C , \& Marchand, G., Eds.). Actes de la séance de la Société préhistorique française de Rennes, 10-11 avril 2014. Société préhistorique française Séances de la Société préhistorique française, (6): 283-319. (in French) ("New approaches of the sea hunter-gatherers in France”) URL: http://www.prehistoire.org/offres/file_inline_src/515/515_P_41148_58512e3e298 f5_14.pdf

Marchand, G., Perrin, T. 2015, Why this revolution? Explaining the major technical shift in Southwestern Europe during the 7th millennium cal. BC, Quaternary International, 428: 73-85. (in English) ("Pourquoi cette révolution ? Explication du changement technique majeur en Europe sud occidentale durant le $7^{\mathrm{e}}$ milléanaire BCE”) doi:10.1016/j.quaint.2015.07.059

Michel, S. 2009, Le Premier Mésolithique du Centre-Ouest : modalités techniques de l'industrie lithique, Bulletin de la Société préhistorique française, 106(4): 715-734. (in French) ("The First Mesolithic of Middle-West : technical modalities of lithic industry") doi:10.3406/bspf.2009.13892

Parent, R. 1967, Le gisement tardenoisien de l'Allée Tortue, à Fère-en-Tardenois (Aisne). Bulletin de la Société préhistorique française, 64(1): 187-208. (in French) ("The 
tardenoisian site of l'Allée Tortue, à Fère-en-Tardenois (Aisn)”)

doi:10.3406/bspf.1967.4111

Pelegrin, J. 1988, Débitage expérimental par pression : du plus petit au plus grand. In: Technologie préhistorique (Tixier, J, Ed.), Notes et Monographies Techniques du CRA, Vol. 25, Edition du CNRS, Paris: p. 37-53. (in French) ("The pressure debitage experiment : from the smallest to the largest”)

Pelegrin, J. 1991, Aspects de démarche expérimentale en technologie lithique. In: 25 ans d'études technologiques en Préhistoire : Bilan et perspectives. XIème rencontres internationales d'Archéologie et d'Histoire d'Antibes. Éditions APDCA (Association pour la promotion et la diffusion des connaissances archéologiques), Juan-les-Pins: p. 57-63. (in French) (“Aspects of the experimental study in lithic technology”)

Pelegrin, J. 2006, Long blade technology in the old World: an experimental approach and some archaeological results. In: Skilled Production and Social Reproduction (Apel, J., \& Knutsson, K., Eds.), Stone Studies Vol. 2, Societas Archaeologica Upsaliensis, Uppsala: p. 37-68. (in English) (“Technologie des grandes lames dans l'ancien monde : une approche expérimentale et des résultats archéologiques”)

Pelegrin, J. 2012, New Experimental Observations for the Characterization of Pressure Blade Production Techniques. In: The Emergence of Pressure Knapping: From Origin to Modern Experimentation (Desrosiers, P. \& Rhamani N., Eds.), Springer-Verlag, New York: p. 465-500. (in English) (“Nouvelles observations expérimentales de la charactérisation des techniques de production des lames à la pression”) doi:10.1007/978-1-4614-2003-3_18

Pelegrin, J. \& Riche, C. 1999, Un réexamen de la série de Bouvante (Drôme) : matières premières lithiques et composantes technologiques. In: Circulations et identités culturelles alpines à la fin de la Préhistoire : matériaux pour une étude. (Programme collectif CIRCALP - 1997/1998) (Beeching, A., Ed.). Travaux du Centre d'Archéologie préhistorique de Valence Vol. 2, Agence Rhône-Alpes pour les sciences humaines, Centre d'Archéologie préhistorique de Valence, Valence: p. 183-195. (in French) (“A new study of Bouvante 'Drôme) : raw material and technology")

Perrin, T. \& Binder, D. 2014, Le Mésolithique à trapèzes et la néolithisation de l'Europe sudoccidentale. In: La transition néolithique en Méditerranée. Actes du colloque Transitions en Méditerranée, ou comment des chasseurs devinrent agriculteurs (Manen, C., Perrin, T., \& Guilaine, J., Eds.), Muséum de Toulouse, 14-15 avril 2011, Éditions Errance et Archives d’Écologie Préhistorique, Arles et Toulouse: p. 271-281. (in French) ("The blade and trapeze mesolithic and the neolithisation in southwestern Europe”)

Perrin, T., Marchand, G., Allard, P., Binder, D., Collina, C., Garcia-Puchol O., Valdeyron, N., 2009, Le second Mésolithique d’Europe occidentale : origine et gradient chronologique. Annales de la Fondation Fyssen, 24: 160-177. (in English) ("the late Mesolithic of Western Europe: origins and chronological stages”)

Perrin, T., Allard, P., Marchand, G., Binder, D., Garcia Puchol, O., Valdeyron, N. sous presse, The late Mesolithic of Western Europe: origins and diffusion of blade and trapeze industries. In: Méso'2010. Proceedings of the Eight International Conference on the Mesolithic in Europe, Santander (ES), Sept. 2010 (Arias, P., Ed.), Éd. Oxbow Books, Oxford, nombre de pages oublié. (in English) ("Le Mésolithique récent de l'Europe occidentale : origine et diffusion des industries à lames et trapèzes”) 
Robert, B., Allard, P., Hamon, C. 2015, Tombe à incinération du Mésolithique à Concevreux (Aisne). Revue archéologique de Picardie, (Hommages à Mariannick Le Bolloch,) 3(4): 15-32. (in French) (“A Mesolithic cremation grave at Concevreux (Aisn)”)

Rozoy, J.-G. 1968, Typologie de l'Épipaléolithique (Mésolithique) franco-belge. Bulletin de la Société préhistorique française, Études et travaux, 65(1): 365-390. (in French) ("Typology of the Epipaleolithic (Mesolithic) in France and Belgium") doi:10.3406/bspf.1968.4157

Rozoy, J.-G. 1978, Les derniers chasseurs : l'Épipaléolithique en France et en Belgique, essai de synthèse. Bulletin de la Société archéologique champenoise (Mémoire), Numéro spécial juin 1978, Société Archéologique Champenoise, Charleville-Mezière, 3 Vol., 1256 p. (in French) ("The last hunter-gatherers : Epipaleolithic in France and Belgium, attempted synthesis”)

Séara, F. \& Bostyn, F. 2009, L’occupation Mésolithique final du site de Lhéry dans la Marne. In: Proceedings of the international congress "Chronology and Evolution in the Mesolithic of NW Europe. Brussels, May 30 - June 12007 (Crombe', P., Van Strydonck, M., Sergant, J., Bats ,M., \& Boudin, M., Eds.), Cambridge Scholar Publishing, Newcastle upon Tyne: p. 767-784. (in French) ("The Late Mesolithic site of Lhery in the Marn”)

Séara, F., Rotillon, S., Cupillard, C. 2002, Campements mésolithiques en Bresse jurassienne ; Choisey et Ruffey-sur-Seille (Jura). Documents d'archéologie française Vol. 92, Maison des sciences de l'homme (MSH), Paris, 344 p. (in French) ("Mesolithic campsites in Bresse jurassienne Choisey et Ruffey-sur-Seille (Jura)”)

Souffi, B. 2004, Le Mésolithique de Haute-Normandie : l'exemple d'Acquigny " l'Onglais » (Eure) et sa contribution à l'étude des gisements mésolithiques de plein-air, BAR International Series Vol. 1307, Archaeopress, Oxford, 208 p. ("The Mesolithic in High Normandy : the example of d'Acquigny « l'Onglais » (Eure) and its contribution to the study of mesolithic open air sites”)

Souffi, B., Guéret C., Leduc C. 2015, Nouvelles données sur le RMS-A et le Mésolithique final dans le nord-est de la France : le site mésolithique de Rémilly-les-Pothées « la Culotte » (Ardennes, France). In: Le second Mésolithique, des Alpes à l'Atlantique (VII ${ }^{\circ}$ - $V^{\circ}$ millénaire). Table ronde internationale de Strasbourg. 2015, Résumé des communications (Jeunesse, C, \& Séara, F., Eds.) Ministère de la Culture (Direction régionale des affaires culturelles d'Alsace, Strasbourg: p. 24-25. (in French) ("New datas for the RMS-A and the final Mesolithic in northwestern France : the mesolithic site of Rémilly-les-Pothées « la Culotte » (Ardennes, France)”).

URL: http://www.misha.fr/sites/5/File/2015nov3.pdf

Thevenin, A. 1998, Le Mésolithique du Centre-Est de la France. Revue Archéologique de l'Est, 49: 87-133. (in French) ("Mesolithic in France Middle East”)

Tixier, J. 1982, Techniques de débitage : Osons ne plus affirmer. Studia Praehistorica Belgica, 2: 13-22. (in French) (“Technique of débitage : let us dare not to affirm”)

Valdeyron, N. 2008, The Mesolithic in France. In: Mesolithic Europe (Bailey, G. \& Spikins, P., Eds). Cambridge University Press, Cambridge: p. 182-202. (in English) ("Le mésolithique en France")

Verjux, C. 2015, Les structures en creux du site mésolithique d'Auneau «le Parc du Château » (Eure-et-Loir). Nouveau bilan et implications concernant le mode de vie des 
dernières populations de chasseurs-collecteurs en Europe. Thèse de Doctorat, Université de Paris I, Panthéon-Sorbonne, Paris, 397 p. (in French) ("The pits of the Mesolithic site at Anneau "le Parc du Château" (Eure-et-Loir). Overview and implications about way of life of the last hunter gatherers in Europe")

Walczak, J. 1998, La question des styles techniques durant le Mésolithique : remarques générales sur le style tardenoisien de Coincy et sur sa «valeur humaine ». Bulletin de la Société préhistorique française, 95(2): 203-220. (in French) ("The question of technic styles during the Mesolithic : général remarks about the tardenoisian style of Coincy and on its human valor”) doi:10.3406/bspf.1998.10768 


\title{
Variability of laminar debitage in the second Mesolithic and early Neolithic in the north of France $\left(7^{\text {th }}\right.$ and $6^{\text {th }}$ millennium BCE)
}

\author{
Pierre Allard \\ CNRS, UMR-7055, Préhistoire et Technologie, Maison de l’Archéologie et de l’Ethnologie, 21 allée de \\ l’Université, 92023, Nanterre Cedex, France. Email: pierre.allard@cnrs.fr
}

\begin{abstract}
:
This paper presents an overview of the knapping techniques of the Second Mesolithic and Early Neolithic during the $7^{\text {th }}$ and $6^{\text {th }}$ millennium in Northern France. This period is characterised by the emergence of new armatures - the trapezes - and of new techniques of prismatic blade production that show discontinuation from the preceding technical traditions. Basically, if we admit that the success of these new technical practices is linked to their intrinsic qualities (higher regularity and parallelism of the blanks, control of the gesture and of the impact point as well as higher productivity), this can explain that the investment into the production focuses on the blanks rather than on the processes of retouch.
\end{abstract}

This paper presents the current state of the data regarding the knapping techniques and their distribution. This analysis is based on the technological approach aiming at characterizing the types of production and the techniques of percussion.

At the current state of analysis, only indirect percussion (punch) knapping technique is observed in the northern and western part of the area. Nonetheless, a more detailed study is still required in order to better describe distinct, very regular bladelet productions made from small-sized cores (eastern part of France). With respect to the geographical extension of the considered area, it can be stated that technological studies are still indigent. The debitage of L'Essart A seems to correspond to the common thread of the Late Mesolithic types of production by indirect percussion. However, some well-documented areas allow the perception of different types of indirect percussion which involves variants independent from the raw material which become apparent in the knapping procedures. This is the case of the the Retzian which is clearly opposed to the Teviecian through the procedures of systematic core preparation by microfacetting but also through the outstanding stylistic unity of the trapeze types. Debitage on the edge of a flake for the creation of blade surfaces seems for instant restricted to the Tardenoisian in a wider sense which means an area extending from the Paris Basin to Belgium.

The chronological dimension is a crucial factor that has to be incorporated into the analysis. With regard to indirect percussion, an expanded chronological framework of the lithic assemblages would permit to understand whether the different variants are synchronous or diachronic. New discoveries gave us a better idea of the beginning of indirect percussion in Paris basin (around $6200 \mathrm{cal} \mathrm{BC}$ according to the dating of the grave of Cuiry-lès-Chaudardes).

The study of this " complex » of trapeze industries encompasses several levels of observation that have to be refined. The phenomenon is considered here globally. But as we have seen, some well defined entities seem to emerge within this complex. These are preliminary results for the moment and an interpretation that makes sense has yet to be made for these different practices. The typological evolution of trapezes and derived triangles has to be incorporated into the results obtained from the technological approach in order to determine whether the stylistic and technological entities can be 
correlated. The precise intersection between the stylistic entities and the technological variants assumed here has to be demonstrated. It will probably be possible to subdivide this complex in order to better understand its evolution.

Keywords: trapeze industry; blade debitage; lithic technology, Mesolithic, Early Neolithic 